\title{
A 2-D Filtering Scheme for Stereo Image Compression Using Sequential Orthogonal Subspace Updating
}

\author{
Sang-Hoon Seo and Mahmood R. Azimi-Sadjadi, Senior Member, IEEE
}

\begin{abstract}
Stereo image compression involves estimating the disparity vectors that represent the amount of binocular parallax. The mismatching problems between the left and right images greatly impact the accuracy of the reconstructed image, and hence the visual effects of the reproduced 3-D image. This paper presents a new method for compensating the mismatching effects in stereo image pairs. This 2-D filtering-based scheme uses a sequential orthogonal subspace updating (SOSU) process to project an image block onto a subset of best-basis vectors. The basis vectors are selected one by one from the neighboring blocks, as well as some typical edge blocks, forming an image-dependent set of basis vectors. This leads to the optimal representation of an image block with fewer coefficients. Simulation results on two different image pairs demonstrate the effectiveness of the SOSU scheme when compared to those of the standard least squares 2-D filtering and the hybrid disparity-compensated discrete cosine transform residual encoding schemes.
\end{abstract}

Index Terms-2-D filtering, orthogonal projection updating, stereo image compression.

\section{INTRODUCTION}

$\mathbf{T}$ HREE-DIMENSIONAL (3-D) video using stereo image sequences is being studied actively due to its capability in providing stereoscopic pictures with high resolution and great sensation of reality. This can be useful in many applications, such as 3DTV, video conferencing, computer games, augmented reality, surgical environments, remote sensing, and robotics. In these applications, it is often necessary to efficiently compress the stereo image sequences by utilizing the characteristics that there are very strong correlation between the right and left images, as well as between the current and previous frames.

Disparity estimation aims at finding the position errors, or the binocular parallaxes, between the points or blocks corresponding to the right and left images of a stereo image pair. This is very similar in nature to the motion estimation used to detect the object displacement in video image sequences. Consequently, many algorithms that are used for motion estimation can also be applicable to the disparity estimation problem. The estimation of disparity vectors, however, needs greater accuracy compared with the estimation of motion vectors. This is due to the fact that human eyes recognize still objects sharper than

Manuscript received January 5, 1998; revised June 26, 2000. This paper was recommended by Associate Editor S.-P. Liou.

The authors are with the Department of Electrical and Computer Engineering, Colorado State University, Fort Collins, CO 80523 USA (e-mail: azimi@engr.colostate.edu).

Publisher Item Identifier S 1051-8215(01)00673-5. moving ones and have higher resolution with 3-D images than 2-D images [1]. The error in estimating disparity vectors can greatly affect the quality of the reconstructed 3-D images. Additionally, the stereo image compression involves mismatching problems between the left and right images due to several factors including reflectivity/illumination differences, deformation of objects, occlusion and noise. These effects have to be compensated for in order to provide good quality reconstructed 3-D images at the receiver.

The standard block-matching method [2] is generally used for the disparity estimation, as well as motion estimation. This simple method performs a translational matching scheme with minimal algorithmic complexity. The disparity-compensated (DC) residual image is typically encoded using the discrete cosine transform (DCT). This is similar to the hybrid motion-compensated DCT scheme, which is currently adopted as the standard for video communications [3], [4] due to its simplicity, low coding overhead requirements, and suitability for hardware implementation. However, this simple scheme suffers from several limitations such as blocking artifacts on the reconstructed images and poor compensation ability for the mismatched areas. The latter is particularly the case for stereo image compression applications.

To provide better compensation ability before the residual encoding, different variants of the block-matching method, namely the generalized block-matching method [5] and variable block-size block-matching method [6], are developed. The generalized block-matching method compensates for the deformation of objects using a spatial transformation. The method in [6], on the other hand, segments the blocks depending on the mean-squared error (MSE) criterion. Blocks with mismatching problems or different depth objects, which usually lead to large MSE, are partitioned into smaller blocks to increase the homogeneity inside the block. The compensation ability of these algorithms is obtained at a price of either a very complicated procedure to get the exact matching transformation parameters [5], or considerable encoding overhead requirements to deal with the mismatching areas [6].

The projection-based filtering schemes in [7]-[13] have recently been developed in order to circumvent the above-mentioned problems and provide better compensation ability. The subspace projection technique [7], [8] for stereo image compression generates a set of orthogonal basis vectors, from four blocks for every image block, using the Gram-Schmidt (GS) orthogonalization procedure. Three out of the four blocks are fixed: one block contains all ones (1's) to compensate for the 
intensity differences while two blocks provide tilt in each of the $x$ and $y$ directions, respectively. The fourth block is the one for which the disparity vector is estimated using the blockmatching method. Although, the orthogonalization process in this method is not computationally demanding (since three blocks are fixed and only one is changing depending on the content of the image block), the fixed blocks may not be adequate to compensate for different types of mismatching problems that can occur. The matching pursuits (MP) algorithm in [9]-[11] expands the motion compensated residual image using an overcomplete dictionary of basis vectors, namely the Gabor functions that remove the grid positioning restrictions in the block-based schemes. MP places virtually no restriction on the choice of the dictionary set. Although Gabor and wave packets [9] have typically been used, it is possible to control which type of image features are encoded well by choosing appropriate dictionary functions that match the shape, scale or frequency of the desired features. The implementation of this iterative nonorthogonal [10], [11] expansion involves six steps: 1) computation of an energy map; 2) finding the best "atom" and its parameters; 3) quantizing the projection coefficient; 4) computing a new residual; 5) encoding position information; and 6) encoding the projection coefficient and accompanying basis function indexes. For Gabor basis, an atom is found by finding the largest inner product along with the corresponding dictionary functions [10]. This step obviously involves performing a large number of inner product operations for each pixel in the search region with high energy. Although, MP has been shown [10] to offer better PSNR than the H.263 standard for image sequence coding, in presence of global motion such as panning and zooming, or when objects leave or enter a scene, this method becomes less effective than the orthogonal transform-based encoding methods like DCT. Consequently, the MP method may not be efficient for compensating such mismatching problems as occlusion, deformation, and even intensity differences in stereo image compression. Moreover, this method is not suitable for block-based filtering due to different support of Gabor [10], [11] and some wave packet [9] basis functions which may span outside the block boundary. The multistage gain/shape vector quantization [12], [13] represents the desired vector with the products of the gain and shape codewords. The shape codebook consists of normalized image blocks. The vector that provides maximum inner product with the desired vector is selected. Then, the gain vector is obtained to encode the inner product value. The difficulty in this method is how to generate optimal multistage codebooks for the whole image and at the same time guarantee good quality reconstructed images.

Although the above-mentioned subspace filtering schemes may offer low encoding overhead, they are not likely to provide the good compensation ability needed in stereo imaging applications. As pointed out before, this is crucial as it directly affects the visual effects of the reproduced 3-D images. In an attempt to solve these problems, we proposed a least-squares (LS)-based 2-D filtering scheme [14], [15]. This approach compensates the mismatching problems by applying the left image to the reference input of a 2-D filter while using the right image as the desired output. To minimize the number of filter coefficients needed for reconstruction, a reduced-order filtering scheme was also introduced which recursively allocates variable filter order until certain quality measure for every reconstructed block is met. The reconstructed images generated based upon the estimated disparity vectors and some principal filter coefficients show the success of the method in removing the mismatching problems. Nonetheless, this scheme requires intensive computation to estimate disparity vectors, owing to the fact that the 2-D filter has to be applied to all the blocks within the search region. In addition, the compensation ability is limited since the filter input blocks are only confined to those blocks inside the support region that is determined by the filter size.

In this paper, a new 2-D filtering scheme using sequential orthogonal subspace updating (SOSU) is introduced which circumvents the limitations of the LS-based 2-D filtering scheme [14], [15] and enhances its compensation ability. The basis vectors are formed from an extended set of neighboring blocks as well as some typical edge blocks for a more flexible and accurate representation. These image-dependent basis vectors are well-matched to the shape and edge structures within an image block. The SOSU uses an iterative approach similar to the MP method in order to find a set of best basis vectors that minimizes the MSE for each block. At every stage of the algorithm, the remaining blocks are orthogonalized using the GS procedure [16] and the residual (or innovation) image is projected onto the orthogonalized subspace and the coefficient corresponding to the largest projection is extracted. The process continues until certain quality measure is satisfied. The main features of this method include: optimal allocation of a variable and image-dependent number of input blocks for each desired block and great compensation capability. These are achieved without the expense of increasing the computational effort.

The organization of this paper is as follows. In Section II, the LS-based 2-D filtering scheme is introduced in the context of orthogonal projection. Section III, presents the idea of SOSU and the derivation of some key equations. Simulation results and comparison with the original LS-based 2-D filtering and hybrid DC-DCT are provided in Section IV. Finally, Section V gives the concluding remarks on this work.

\section{LEAST-SQUARES FILTERING USING ORTHOGONAL PROJECTION UPDATING}

To account for the effects of mismatching problems due to illumination differences, object occlusion or deformation in the stereo image pair, a 2-D filter is used in conjunction with the block-matching method. Fig. 1 depicts the block diagram of the system. In this system, the mismatching effects are compensated for by applying the right image, in the stereo image pair, as the desired image and the left image as the reference input image of the 2-D filter. The right image is partitioned into nonoverlapping blocks of size $K \times L$, where each block is used as the desired block that needs to be reconstructed at the receiver. For every block in the right image, a search region is chosen in the left image to estimate the disparity vector. To avoid filtering all the blocks inside the search region [14], [15], the block matching is first applied to estimate the disparity 


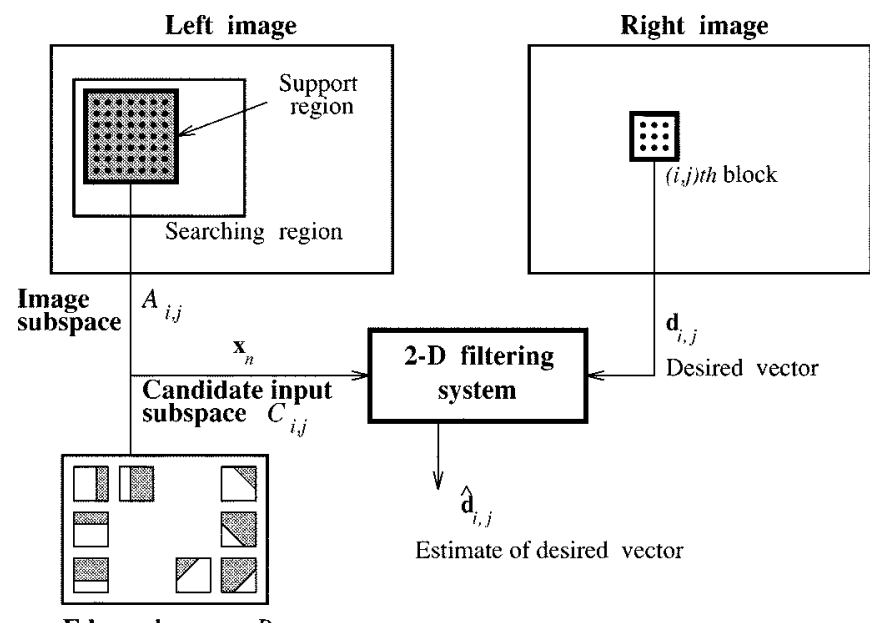

Fig. 1. 2-D LS-based filtering scheme for stereo image compression.

vector. The filter support region is then confined to a region of size $\left(M_{1}+K-1\right) \times\left(N_{1}+L-1\right)$ within the search region. This leads to $M_{1} N_{1}$ blocks within the support region. For the $(i, j)$ th desired (right) image block $\mathbf{d}_{i, j}$ of size $K \times L$, these blocks form an "image subspace" $A_{i, j}$, with $M_{1} N_{1}$ basis vectors, i.e., $A_{i, j}=\left[\begin{array}{lllll}\mathbf{x}_{1} & \mathbf{x}_{2} & \mathrm{x}_{3} & \ldots & \mathrm{x}_{M_{1} N_{1}}\end{array}\right]$, where each block $\mathbf{x}_{n}$ of size $K \times L$ in the support region is arranged into a column vector of size $K L \times 1$. To provide better compensation ability for the edges and boundaries of the objects, different possible edge blocks are also included to form the "edge subspace", $B$, i.e., $B=\left[\begin{array}{llll}\mathbf{x}_{M_{1} N_{1}+1} & \mathbf{x}_{M_{1} N_{1}+2} & \ldots & \mathbf{x}_{M N}\end{array}\right]$. The 62 edge blocks typically used for image/video coding [17] are shown in Fig. 2. These two sets form the entire space $C_{i, j}=A_{i, j} \cup B$, with $M N$ different basis vectors. Clearly, this space in not orthogonal. However, an image representation space does not have to be orthogonal for it to provide optimum performance for a particular task [10]. In this application, the selected subspaces are found to be optimal as they are well-matched to the shape features and spatial content in the image blocks. The desired block $\mathbf{d}_{i, j}$ can be optimally represented in this space by finding the projections of this block on a few principal basis vectors with the largest contributions. The projection coefficients can then be used to reconstruct the desired image satisfying certain fidelity criterion, e.g., PSNR. In this paper, this is accomplished by using the SOSU scheme which is similar in nature to the MP and the order updating process in adaptive transversal filters [18], [19]. The latter approach has been adopted here since it is more suited in providing insight into the filtering process and further leads to a simple sequential orthogonalization procedure.

In the sequel, for notational convenience, the block index $(i, j)$ is suppressed from the equations, since the same process is repeated for each block. In order to implement an order update subspace-based filtering that finds the best set of basis blocks satisfying a predefined fidelity criterion, the orthogonal projection updating [18], [19] is employed. Let us assume that for the desired output block $\mathbf{d}$ in the right image and a chosen candidate input block or basis vector $\mathbf{x}_{n}$ from space $C$, we would like to find the optimal transversal filter coefficient to minimize the
MSE. From the adaptive filter theory [20], this solution corresponds to the LS solution given by

$$
a_{\mathbf{x}_{n}}:=\mathbf{K}_{\mathbf{x}_{n}}^{T} \mathbf{d}
$$

where the matrix $\mathbf{K}_{\mathbf{x}_{n}}$ is called the "transversal filter operator" [18], [21] defined, for the candidate input block, $\mathbf{x}_{n}$, as

$$
\mathbf{K}_{\mathbf{x}_{n}} \triangleq \mathbf{x}_{n}\left(\mathbf{x}_{n}^{T} \mathbf{x}_{n}\right)^{-1} \text {. }
$$

In this case, the actual filter output can be written as

$$
\hat{\mathbf{d}}_{\mathbf{x}_{n}}=a_{\mathbf{x}_{n}} \mathbf{x}_{n}
$$

or alternatively

$$
\hat{\mathbf{d}}_{\mathbf{x}_{n}}=\mathbf{P}_{\mathbf{x}_{n}} \mathbf{d}
$$

where

$$
\mathbf{P}_{\mathbf{x}_{n}} \triangleq \mathbf{x}_{n}\left(\mathbf{x}_{n}^{T} \mathbf{x}_{n}\right)^{-1} \mathbf{x}_{n}^{T}=\mathbf{K}_{\mathbf{x}_{n}} \mathbf{x}_{n}^{T}
$$

is the "projection matrix" [19], [20] associated with $\mathbf{x}_{n}$ basis vector. The error vector in this estimation is

$$
\mathbf{e}_{\mathbf{x}_{n}}=\mathbf{d}-\hat{\mathbf{d}}_{\mathbf{x}_{n}}=\mathbf{d}-\mathbf{P}_{\mathbf{x}_{n}} \mathbf{d}=\mathbf{P}_{\mathbf{x}_{n}}^{\perp} \mathbf{d}
$$

where $\mathbf{P}_{\mathbf{x}_{n}}^{\perp} \triangleq \mathbf{I}-\mathbf{P}_{\mathbf{x}_{n}}$ is called the "orthogonal complement" of the projection matrix $\mathbf{P}_{\mathbf{x}_{n 2}}$. The above result implies that the best estimate for $\mathbf{d}$ (based upon the chosen input block $\mathbf{x}_{n}$ ) that minimizes the sum squared of the error $\mathbf{e}_{\mathbf{x}_{12}}$ is the projection of the vector $\mathbf{d}$ onto a subspace spanned by the column vector $\mathbf{x}_{n}$, and further, $\mathbf{e}_{\mathbf{x}_{n 2}}=\mathbf{P}_{\mathbf{x}_{12}}^{\perp} \mathbf{d}$ is the component which is orthogonal to this subspace. This is the orthogonality principle that is used throughout this paper.

To find the first best or principal block of input data $\mathbf{x}_{n_{1}}$, the sum squared error is computed for all the basis vectors and the one that provides the minimum is then selected, i.e.,

$$
\mathbf{x}_{n_{1}}=\arg \min _{\mathbf{x}_{n} \in C} \mathbf{e}_{\mathbf{x}_{n}}^{T} \mathbf{e}_{\mathbf{x}_{n}}=\arg \min _{\mathbf{x}_{n} \in C} \mathbf{d}^{T} \mathbf{P}_{\mathbf{x}_{n}}^{\perp} \mathbf{d} .
$$

Note that $\mathbf{P}_{\mathbf{x}_{n}} \mathbf{P}_{\mathbf{x}_{n}}=\mathbf{P}_{\mathbf{x}_{n}}$. Then, the corresponding projection coefficient $a_{1}$ and error vector $\mathbf{e}_{1}$ are computed using (1) and (6), respectively.

Now, if the estimate of the filter output, $\hat{\mathbf{d}}_{1}=\mathbf{P}_{\mathbf{x}_{n_{1}}} \mathbf{d}$, using this block of input data does not satisfy the predefined fidelity criterion, another block is selected from space $C$ and appended to the column space of $\mathbf{x}_{n_{1}}$ to generate the subspace $\left\{\mathbf{x}_{n_{1}}, \mathbf{x}_{n}\right\}$. Using the orthogonal projection updating" [19], [20], $\mathbf{K}_{\mathbf{x}_{n_{1}}, \mathbf{x}_{n}}$ can be expanded in terms of $\mathbf{K}_{\mathbf{x}_{n_{1}}}$ and a "new part" which corresponds to the component of $\mathbf{x}_{n}$ orthogonal to $\mathbf{x}_{n_{1}}$, i.e.,

$$
\begin{aligned}
\mathbf{K}_{\mathbf{x}_{n_{1}}, \mathbf{x}_{n}}= & {\left[\begin{array}{ll}
\mathbf{K}_{\mathbf{x}_{n_{1}}} & \mathbf{0}
\end{array}\right]+\mathbf{P}_{\mathbf{x}_{n_{1}}}^{\perp} \mathbf{x}_{n}\left(\mathbf{x}_{n}^{T} \mathbf{P}_{\mathbf{x}_{n_{1}}}^{\perp} \mathbf{x}_{n}\right)^{-1} } \\
& \times\left[\begin{array}{ll}
-\mathbf{x}_{n}^{T} \mathbf{K}_{\mathbf{x}_{n_{1}}} & 1
\end{array}\right]
\end{aligned}
$$

where 0 represents a null vector of size $K L \times 1$. The order updated projection matrix can be represented by

$$
\mathbf{P}_{\mathbf{x}_{n_{1}}, \mathbf{x}_{n}}=\mathbf{K}_{\mathbf{x}_{n_{1}}, \mathbf{x}_{n}}\left[\begin{array}{ll}
\mathbf{x}_{n_{1}} & \mathbf{x}_{n}
\end{array}\right]^{T} .
$$




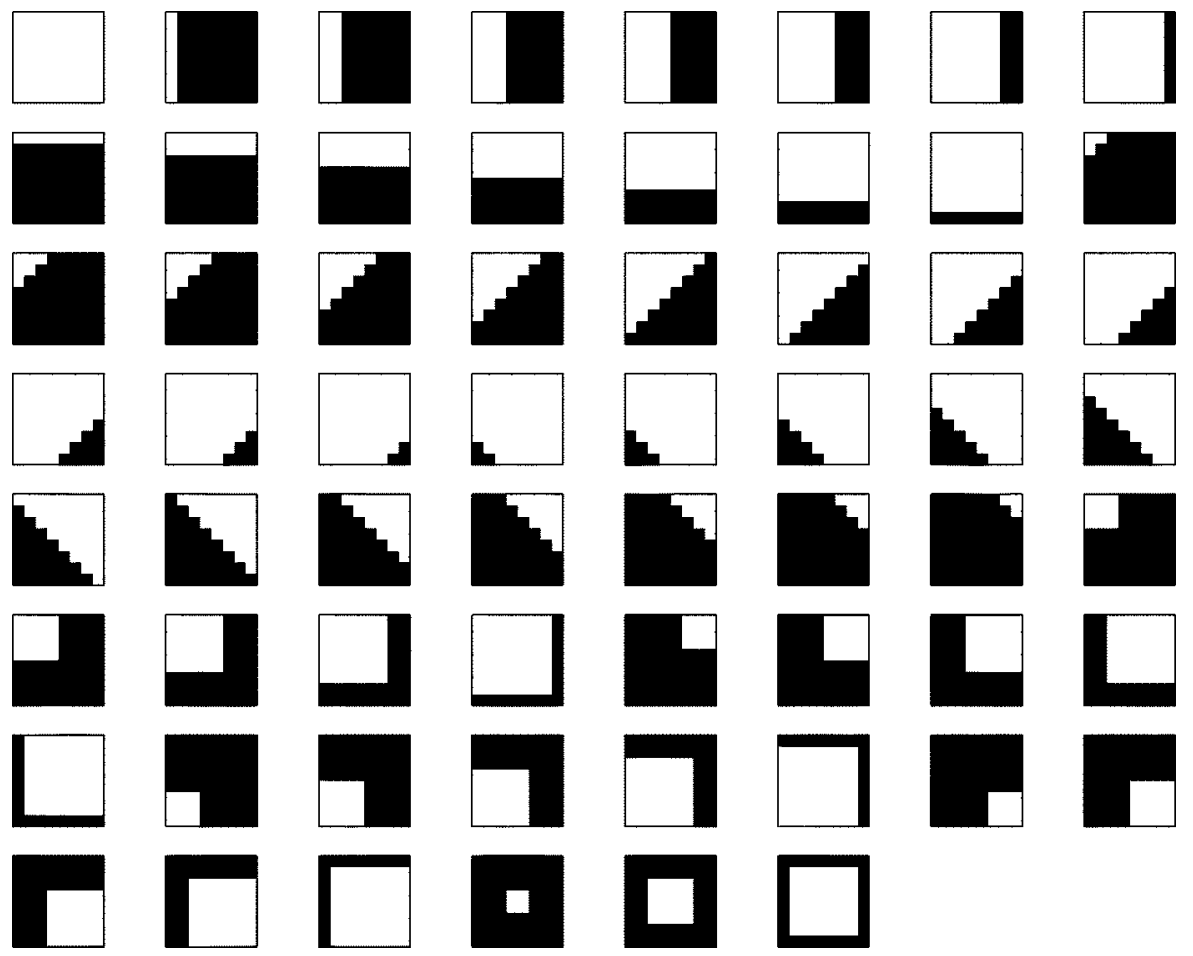

Fig. 2. Typical edge blocks forming subspace $B$.

The error vector in this case is $\mathbf{e}_{\mathbf{x}_{n_{1}}, \mathbf{x}_{n 2}}=\mathbf{P}_{\mathbf{x}_{n_{1}}, \mathbf{x}_{n}}^{\perp} \mathbf{d}$. The second best block of the input data $\mathbf{x}_{n_{2}}$ is then selected to minimize the sum squared error or residual, i.e.,

$$
\mathbf{x}_{n_{2}}=\arg \min _{\mathbf{x}_{n} \in C} \mathbf{e}_{\mathbf{x}_{n_{1}}, \mathbf{x}_{n}}^{T} \mathbf{e}_{\mathbf{x}_{n_{1}}, \mathbf{x}_{n}}=\arg \min _{\mathbf{x}_{n} \in C} \mathbf{d}^{T} \mathbf{P}_{\mathbf{x}_{n_{1}}, \mathbf{x}_{n}}^{\perp} \mathbf{d} .
$$

The projection coefficients and the error vector based on this new input subspace $\left\{\mathbf{x}_{n_{1}}, \mathbf{x}_{n_{2}}\right\}$ are obtained using $\left[\begin{array}{ll}a_{1} & a_{2}\end{array}\right]^{T}=$ $\mathbf{K}_{\mathbf{x}_{n_{1}}, \mathbf{x}_{n_{2}}}^{T} \mathbf{d}$ and $\mathbf{e}_{2}=\mathbf{P}_{\mathbf{x}_{n_{1}}, \mathbf{x}_{n_{2}}}^{\perp} \mathbf{d}$, respectively. The estimate of the desired block $\mathbf{d}$ is given by $\hat{\mathbf{d}}_{2}=\mathbf{P}_{\mathbf{x}_{n_{1}}, \mathbf{x}_{n_{2}}} \mathbf{d}$. This process continues until this estimate satisfies the predefined quality criterion or until the input data subspace contains the predefined maximum number of blocks or basis vectors from space $C$.

Comparing to the LS-based 2-D filtering scheme in [14], [15], that uses the normal equation to estimate the optimal filter weights, this scheme has certain advantages. Unlike the LS-based 2-D filtering, the support region of the input data is not limited by the filter size and can be extended. Additionally, all the processing is performed using matrix-vector operations (instead of matrix manipulations) without any matrix inversion operation which may lead to singularity problems. The LS filtering using the orthogonal projection updating, however, has some limitations. The transversal filter operator in (8) and projection operator in (9) need to be updated whenever the order of input data subspace is increased. In addition, all the filter coefficients need to be calculated again. The sizes of the transversal filter operator and projection operator matrices become increasingly large leading to computational problems.

In the following section, a new SOSU filtering scheme is proposed that circumvents the above-mentioned limitations of the orthogonal projection updating scheme.

\section{A New 2-D FiLtering Scheme Using SOSU}

To see the connection between the previous orthogonal projection-updating scheme and the proposed SOSU scheme, let us assume that $\mathbf{x}_{n_{2}}$ is orthogonal to $\mathbf{x}_{n_{1}}$, i.e., $\mathbf{x}_{n_{2}}^{T} \mathbf{x}_{n_{1}}=0$. In this case, using the properties $\mathbf{P}_{\mathbf{x}_{n_{1}}}^{\perp} \mathbf{x}_{n_{2}}=\mathbf{x}_{n_{2}}$ and $\mathbf{x}_{n_{2}}^{T} \mathbf{K}_{\mathbf{x}_{n_{1}}}=0$, it can easily be shown that the updating rule in (8) reduces to

$$
\mathbf{K}_{\mathbf{x}_{n_{1}}, \mathbf{x}_{n_{2}}}=\left[\begin{array}{ll}
\mathbf{K}_{\mathbf{x}_{n_{1}}} & \mathbf{K}_{\mathbf{x}_{n_{2}}}
\end{array}\right] .
$$

That is, the transversal filter operator for the appended subspace is expressed in terms of the individual filter operators $\mathbf{K}_{\mathbf{x}_{n_{1}}}$ and $\mathbf{K}_{\mathbf{x}_{n_{2}}}$. Consequently, the projection operator can simply be written as the sum of the two projection matrices, i.e., $\mathbf{P}_{\mathbf{x}_{n_{1}}, \mathbf{x}_{n_{2}}}=\mathbf{P}_{\mathbf{x}_{n_{1}}}+\mathbf{P}_{\mathbf{x}_{n_{2}}}$. The projection coefficients $a_{1}$ and $a_{2}$ are obtained using (1) and (11) as

$$
\begin{aligned}
{\left[\begin{array}{ll}
a_{1} & a_{2}
\end{array}\right]^{T} } & =\mathbf{K}_{\mathbf{x}_{n_{1}}, \mathbf{x}_{n_{2}}}^{T} \mathbf{d} \\
& =\left[\begin{array}{ll}
\mathbf{K}_{\mathbf{x}_{n_{1}}}^{T} \mathbf{d} & \left(\mathbf{x}_{n_{2}}^{T} \mathbf{P}_{\mathbf{x}_{n_{1}}}^{\perp} \mathbf{x}_{n_{2}}\right)^{-1} \mathbf{x}_{n_{2}}^{T} \mathbf{P}_{\mathbf{x}_{n_{1}}}^{\perp} \mathbf{d}
\end{array}\right]^{T} \\
& =\left[\begin{array}{ll}
a_{1} & \frac{\mathbf{x}_{n_{2}}^{T} \mathbf{e}_{1}}{\mathbf{x}_{n_{2}}^{T} \mathbf{x}_{n_{2}}}
\end{array}\right]^{T} \\
& =\left[\begin{array}{ll}
a_{1} & \frac{\mathbf{x}_{n_{2}}^{T} \mathbf{d}}{\mathbf{x}_{n_{2}}^{T} \mathbf{x}_{n_{2}}}
\end{array}\right]^{T}
\end{aligned}
$$

The error vector in this case becomes

$$
\begin{aligned}
\mathbf{e}_{2} & =\mathbf{P}_{\mathbf{x}_{n_{1}}, \mathbf{x}_{n_{2}}}^{\perp} \mathbf{d} \\
& =\mathbf{d}-\mathbf{P}_{\mathbf{x}_{n_{1}}, \mathbf{x}_{n_{2}}} \mathbf{d} \\
& =\mathbf{d}-\mathbf{P}_{\mathbf{x}_{n_{1}}} \mathbf{d}-\mathbf{P}_{\mathbf{x}_{n_{2}}} \mathbf{d} \\
& =\mathbf{d}-a_{1} \mathbf{x}_{n_{1}}-a_{2} \mathbf{x}_{n_{2}} \\
& =\mathbf{e}_{1}-a_{2} \mathbf{x}_{n_{2}} .
\end{aligned}
$$


It is interesting to note that, in this case, where $\mathrm{x}_{n_{1}}$ and $\mathrm{x}_{n_{2}}$ are orthogonal, all the operations can be carried out separately and there is no need to recalculate the first coefficient $a_{1}$ when computing the second one. This important feature greatly simplifies the computation of the filter coefficients. Additionally, all the formulations can be done using vector inner products, hence significantly reducing the computational effort.

The idea of orthogonalizing the candidate input blocks prior to the orthogonal projection updating can thus be utilized to arrive at a very simple SOSU scheme. The best basis vectors are obtained one by one in a sequential manner by minimizing the MSE between the desired block and the estimated block using the selected subspace. This SOSU scheme uses the GS orthogonalization procedure [16]. The first input vector is simply obtained by performing

$$
\mathbf{x}_{n_{1}}=\arg \max _{\mathbf{x}_{n} \in C}\left|\frac{\mathbf{d}^{T} \mathbf{x}_{n}}{\mathbf{x}_{n}^{T} \mathbf{x}_{n}}\right|
$$

The corresponding projection coefficient (first one) is then found using

$$
a_{1}=\frac{\mathbf{d}^{T} \mathbf{x}_{n_{1}}}{\mathbf{x}_{n_{1}}^{T} \mathbf{x}_{n_{1}}}
$$

The error vector for this subspace is given by

$$
\mathbf{e}_{1}=\mathbf{d}-a_{1} \mathbf{x}_{n_{1}} .
$$

Note that (15) and (16) are the same as (1) and (6) in the previous section. After the first block or basis vector is selected, the remaining vectors in $C$ are orthogonalized to the first basis vector, $\mathrm{x}_{n_{1}}$, using

$$
\mathbf{x}_{n}^{(1)}=\mathbf{x}_{n}-\frac{\mathbf{x}_{n}^{T} \mathbf{x}_{n_{1}}}{\mathbf{x}_{n_{1}}^{T} \mathbf{x}_{n_{1}}} \mathbf{x}_{n_{1}}, \quad \mathbf{x}_{n}^{(1)} \in C_{i, j}^{(1)}, \quad \forall n \neq n_{1}
$$

where $\mathbf{x}_{n}^{(1)}$ contains the new information not included in $\mathbf{x}_{n_{1}}$, i.e., $\mathbf{x}_{n}^{(1)}=\mathbf{P}_{\mathbf{x}_{n_{1}}}^{\perp} \mathbf{x}_{n}$. This can be used to select the next most effective basis vector to approximate the error signal $\mathbf{e}_{1}$. This process continues and the $t$ th input or basis vector is chosen based on the vectors of subspace $\mathbf{x}_{n}^{(t-1)} \in C_{i, j}^{(t-1)}$, using

$$
\mathbf{x}_{n_{t}}=\arg \max _{\mathbf{x}_{n}^{(t-1)} \in C_{i, j}^{(t-1)}}\left|\frac{\mathbf{e}_{t-1}^{T} \mathbf{x}_{n}^{(t-1)}}{\mathbf{x}_{n}^{(t-1)^{T}} \mathbf{x}_{n}^{(t-1)}}\right| \text {. }
$$

The projection coefficient corresponding to $\mathbf{x}_{n_{t}}$ is similarly determined using

$$
a_{t}=\frac{\mathbf{e}_{t-1}^{T} \mathbf{x}_{n_{t}}}{\mathbf{x}_{n_{t}}^{T} \mathbf{x}_{n_{t}}}
$$

The residual error vector is then given by

$$
\mathbf{e}_{t}=\mathbf{e}_{t-1}-a_{t} \mathbf{x}_{n_{t}}
$$

Note that (19) and (20) are nothing but (12) and (13) for $t=2$. The remaining vectors in $C_{i, j}^{(t-1)}$ are then orthogonalized to this newly chosen basis vector $\mathbf{x}_{n_{t}}$ using

$$
\mathbf{x}_{n}^{(t)}=\mathbf{x}_{n}^{(t-1)}-\frac{\mathbf{x}_{n}^{(t-1)^{T}} \mathbf{x}_{n_{t}}}{\mathbf{x}_{n_{t}}{ }^{T} \mathbf{x}_{n_{t}}} \mathbf{x}_{n_{t}}, \quad \mathbf{x}_{n}^{(t)} \in C_{i, j}^{(t)}, \quad \forall n \neq n_{t} .
$$

This procedure continues iteratively until the quality of the reconstructed image block satisfies the predefined quality criterion. The desired block is then reconstructed using

$$
\hat{\mathbf{d}}_{\mathbf{T}}=\sum_{t=1}^{T} a_{t} \mathbf{x}_{n_{t}}
$$

where the number of the selected basis vectors, $T$, is variable depending on the spatial content of each desired block. This leads to a flexible and accurate representation of a desired block using the smallest possible number of basis vectors well matched to the edge and shape characteristics within the desired block. The effectiveness of the proposed scheme for stereo image compression application will be demonstrated in the subsequent section on two different stereo image pairs.

\section{SIMULATION RESUlTS AND COMPARISON}

In this section, the performance of the proposed SOSU scheme for compensating the mismatching effects in stereo image compression is compared to those of the 2-D LS-based filtering [14], [15] and the hybrid DC-DCT [22] schemes. The reason for choosing these schemes for the benchmarking has to do with the fact that the 2-D LS-based filtering in [14], [15] is similar in nature to the proposed scheme, and the hybrid DCT-based method is widely used for motion compensated encoding. The MP method in [10] was not used in this benchmarking owing to the reasons outlined in the Introduction. Two different stereo image pairs were used in this benchmark. The first pair involves several obvious mismatching problems ideal to test the compensation ability of the algorithms. The other is a real stereo pair acquired from the NHK, Japanese Broadcasting Corporation, to demonstrate the performance of our proposed method for 3DTV compression applications. The performance of the reconstructed image is determined based upon the PSNR, i.e.,

$$
\mathrm{PSNR}=10 \log _{10} \frac{255^{2}}{\sigma_{(D-\hat{D})}^{2}}
$$

where $\sigma_{(D-\hat{D})}^{2}$ is the variance of the difference or error image between the original right image $D$ and the reconstructed right image $\hat{D}$. The quality of each reconstructed image block is represented using the energy of the error block between the original and the reconstructed blocks. In practice, a quality measure is decided in terms of the block PSNR (PSNR block $_{\text {) }}$, i.e.,

$$
\mathrm{PSNR}_{\text {block }}=10 \log _{10} \frac{255^{2}}{\mathbf{e}_{t}^{T} \mathbf{e}_{t} / K L}
$$




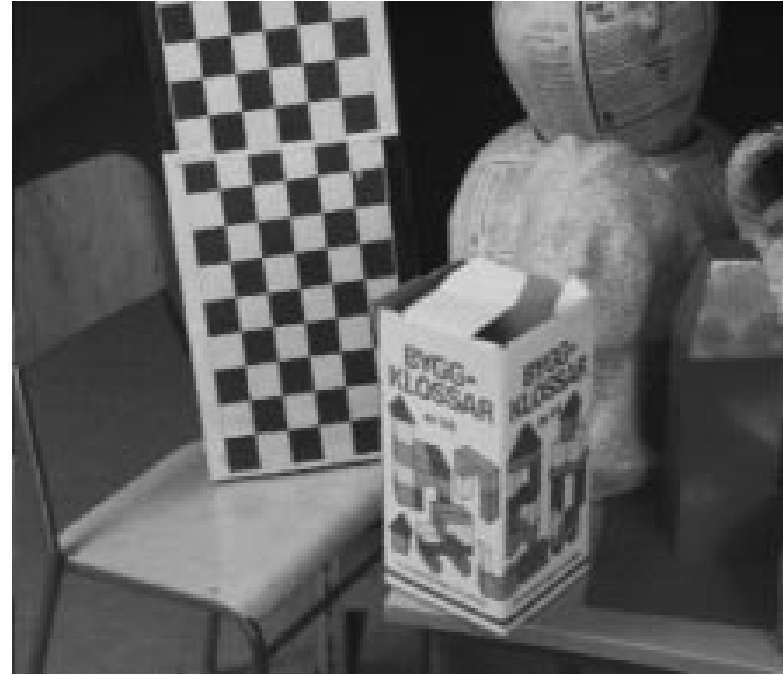

(a)

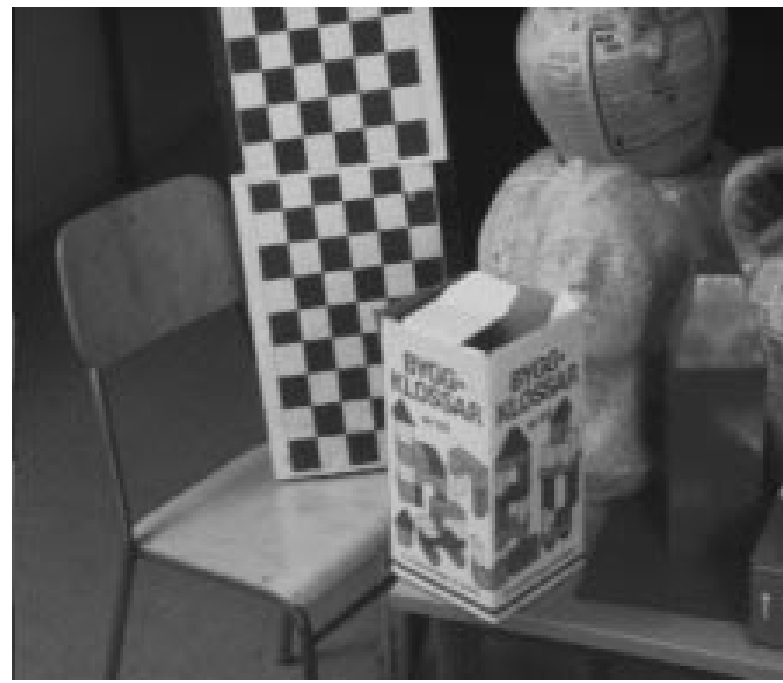

(b)

Fig. 3. Original test stereo image pair "Chair." (a) Original left image (b) Original right image.

where $\mathbf{e}_{t}^{T} \mathbf{e}_{t} / K L$ represents the mean-squared value of the error block which varies from block to block. The MSE is used to account for the intensity mismatching problems between two blocks instead of the variance.

\section{A. Simulation Results on a Test Stereo Image Pair}

The proposed SOSU scheme was first implemented on a test stereo image pair "Chair," shown in Fig. 3(a) and (b). The size of each image is $280 \times 320$ with 256 grey levels. This image pair is very good for testing the SOSU algorithm, since it involves different mismatching problems such as occlusion, reflectivity differences, and deformation of the objects. The right image is used as the desired image and the left image as the reference input image of the filter. The block size was chosen to be $8 \times 8$. The search region was defined as follows: left margin 48 , right margin 8 , upper margin 8 , and lower margin 8 . Note that more margin was given to the left side in the left image since an object in the left image is typically shifted to the left side compared to the right image.

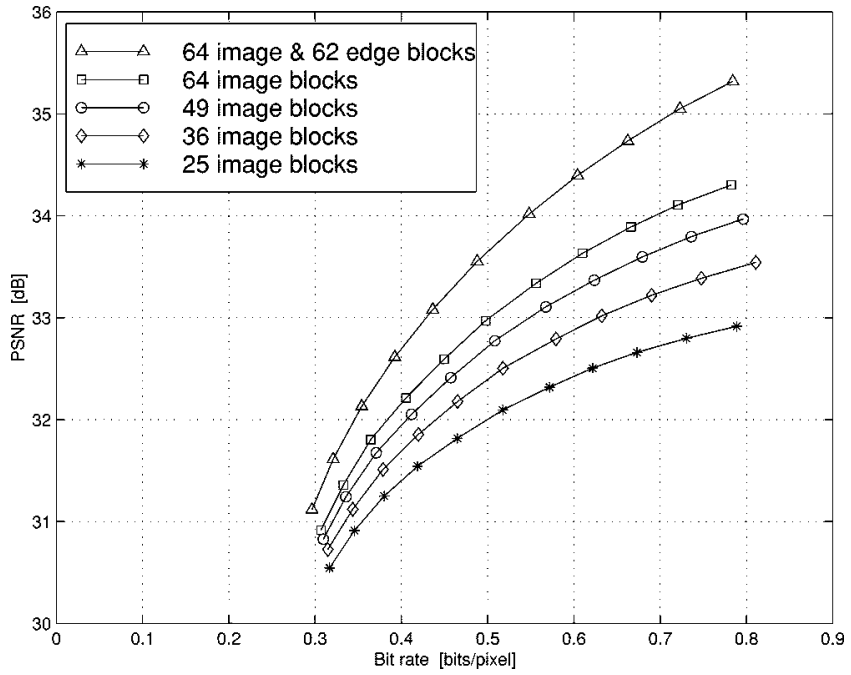

(a)

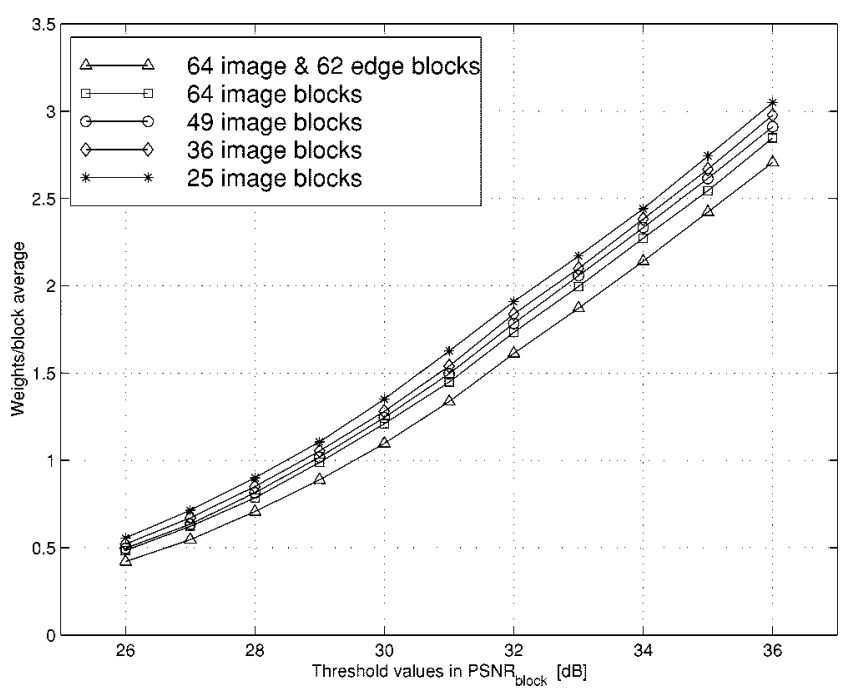

(b)

Fig. 4. Performance of SOSU-PSNR versus bit rate, and quality measures versus average weights/block for the stereo image pair "Chair." (a) PSNR versus bit rate. (b) Quality measure PSNR block $_{\text {: }}$ versus average weights/block.

The disparity vector is first estimated using the standard block-matching method. The support region of the filter input is then confined to the neighborhood around the block at which the disparity vector is found. The support region for the filter input data includes 64 candidate image blocks where each $8 \times 8$ block is arranged into a column vector of size $64 \times 1$. As pointed out before, the proposed SOSU scheme selects the best basis input vectors one by one in order of importance determined based upon the magnitude of the projection coefficient between the desired vector and the candidate input or basis vector. The chosen input basis vectors and their corresponding projection coefficients were then encoded and used to estimate the desired vector at the receiver.

In all the experiments for this image pair, the encoding and bit allocation strategies were as follows. The search region in this case was of size $57 \times 17=969$ pixels. Thus, as far as the disparity vector encoding is concerned, the hybrid DC-DCT method (DDM) and SOSU require 10 bits, since they both use block-matching for disparity estimation, while 


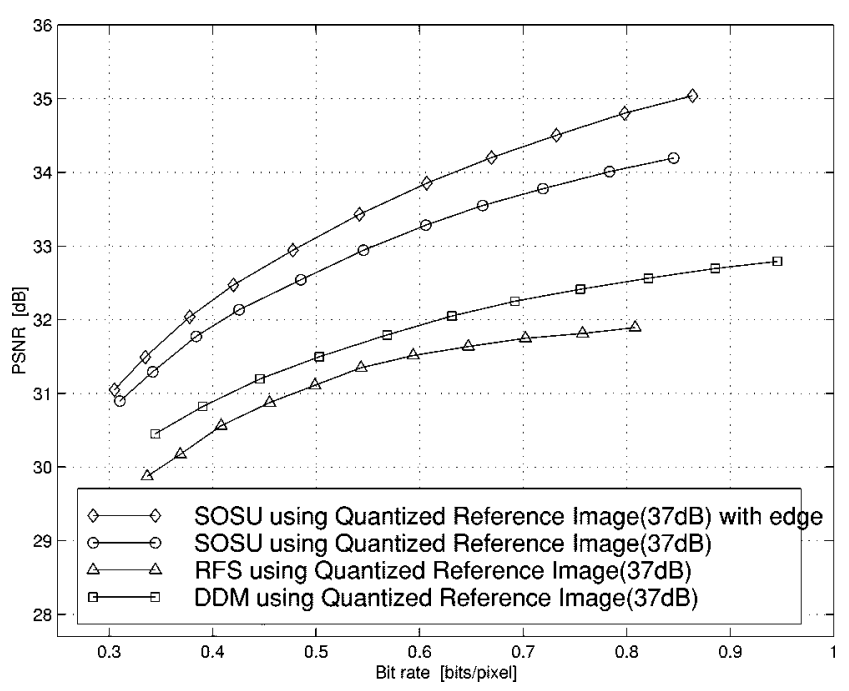

(a)

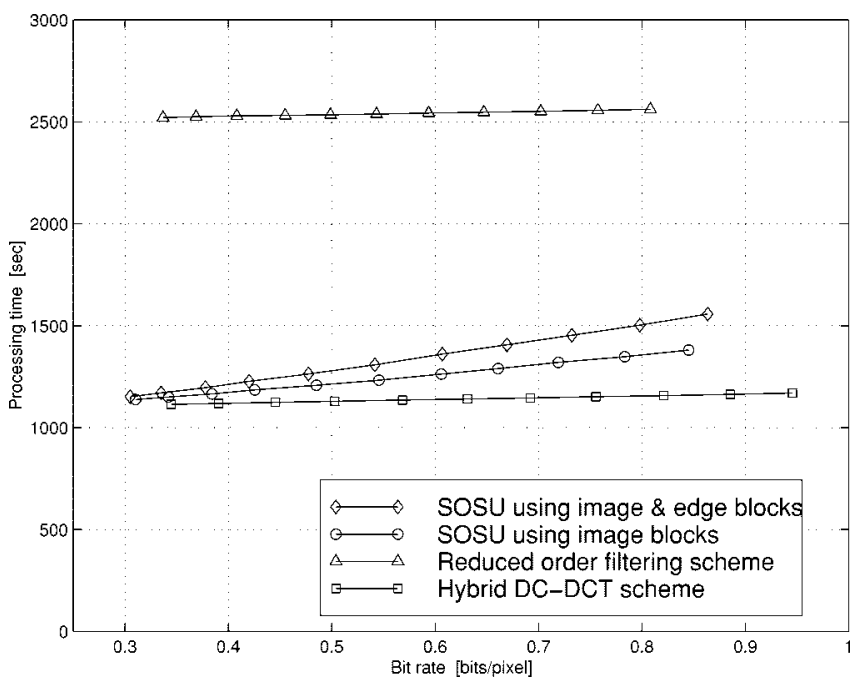

(b)

Fig. 5. Performance comparison of the SOSU scheme with the reduced-order filtering and hybrid DC-DCT schemes on the stereo image pair "Chair." (a) PSNR versus bit rate for different schemes. (b) Processing time versus bit rate for different schemes.

TABLE I

MaXimum, Minimum, AND VARIANCE VAlues of THE ERROR IMAges USING DIFFERENT SCHEMES FOR THE STEREO IMAGE PAIR "CHAIR"

\begin{tabular}{c||c|c|c}
\hline Schemes & Max. value & Min. value & Variance \\
\hline \hline Block-matching & 0.4706 & -0.5255 & 0.00160 \\
\hline Hybrid DC-DCT & 0.2723 & -0.2950 & 0.00057 \\
\hline Reduced order filtering & 0.4472 & -0.3975 & 0.00065 \\
\hline SOSU with image blocks & 0.2444 & -0.2772 & 0.00042 \\
\hline SOSU with image \& edge blocks & 0.2820 & -0.1978 & 0.00035 \\
\hline
\end{tabular}

the reduced-order filtering scheme (RFS) needs 6 bits, since it moves by the window size $(4 \times 4)$. To encode the locations of the basis blocks, the DDM needs 6 bits (64 basis), while SOSU requires 6 bits for the case of no edge block and 7 bits when edge blocks are also included ( $64+62$ basis vectors). The RFS would require only 4 bits to encode the 16 blocks in the support region. To encode the total number of coefficients, all the schemes need 3 bits, since they use maximum of 7 basis blocks to represent an image block. All the filter weights and

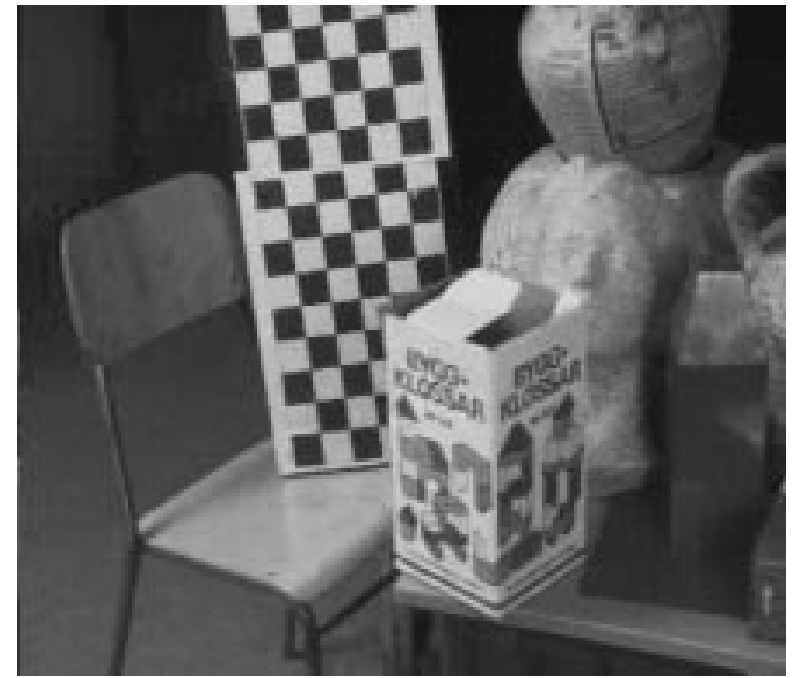

(a)

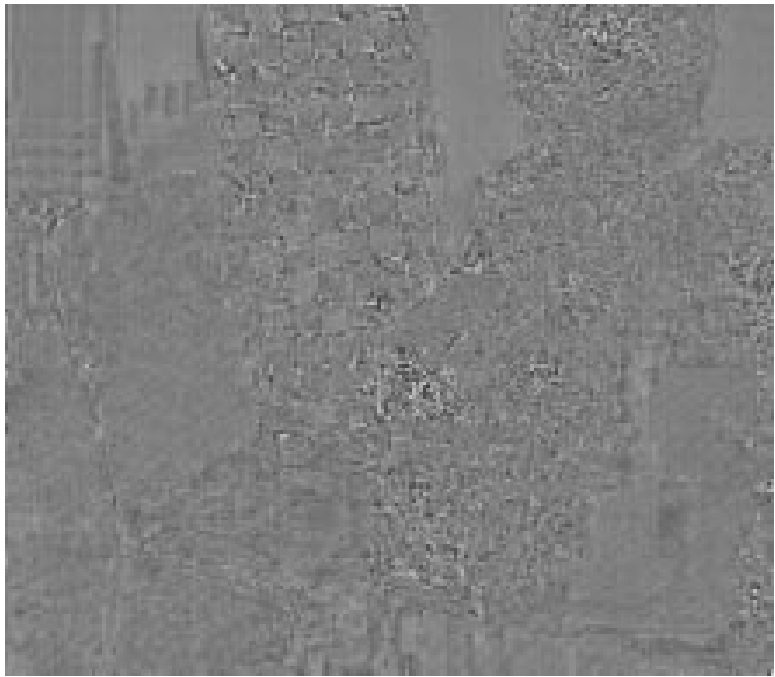

(b)

Fig. 6. Reconstructed and error images using the hybrid DC-DCT scheme at 0.76 bpp on the stereo image pair "Chair." (a) Reconstructed image using hybrid DC-DCT scheme (32.41 dB, 0.76 bpp). (b) Error image using hybrid DC-DCT scheme.

coefficients were encoded using the Lloyd-Max 8-bit quantizer [23].

First, the number of candidate input blocks or basis vectors, determined by the size of the filter support region, was changed to examine it effects on the performance of the SOSU scheme. Fig. 4(a) represents the plot of the PSNR versus bit rate of the reconstructed images for different number of candidate input blocks ranging from 25 to 64 . The last case involved using 64 image blocks and 62 edge blocks as the possible candidate basis vectors in $C$. The SOSU scheme allocates different types and number of input basis vectors to different desired blocks to satisfy the predefined quality measure. Simulations were performed using different quality measure values ranging from

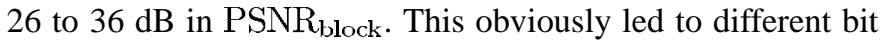
rates to satisfy the fidelity criterion. As can be seen in Fig. 4(a), the PSNR versus bit-rate performance of the reconstructed images is substantially improved as the number of candidate input blocks or basis vectors is increased. The edge blocks 


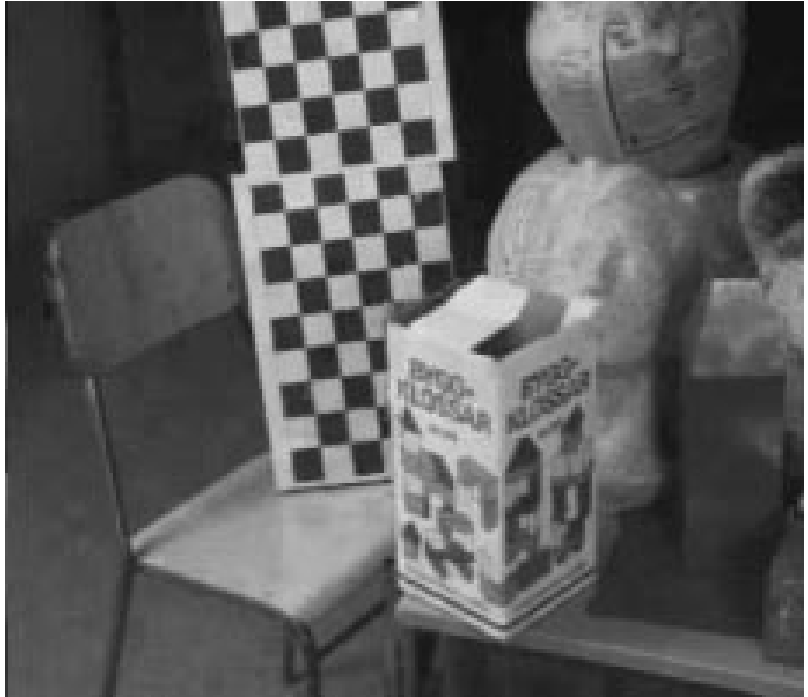

(a)

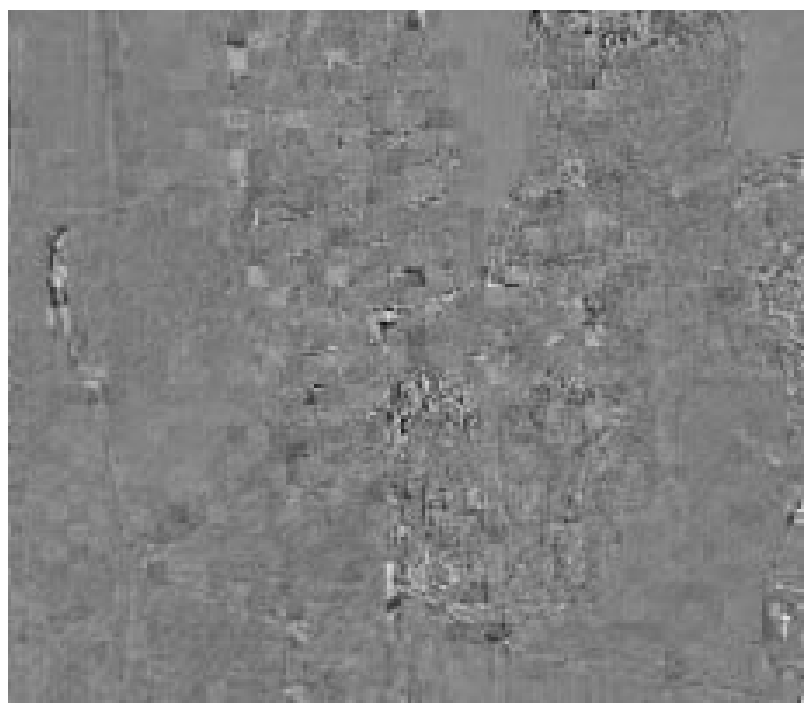

(b)

Fig. 7. Reconstructed and error images using the reduced-order filtering scheme at 0.76 bpp on the stereo image pair "Chair." (a) Reconstructed image using the reduced-ordering filtering scheme (31.81 dB, $0.76 \mathrm{bpp})$. (b) Error image using the reduced-order filtering scheme.

do, indeed, contribute toward improving the performance. Fig. 4(b) reveals an interesting observation that the number of basis vectors, selected for reconstruction satisfying the fidelity measure, gets smaller as the number of candidate input blocks increases. This is due to the fact that including more candidate input blocks in space $C$ enables one to select the most appropriate basis vectors, hence decreasing the number of input basis needed to satisfy the prespecified quality measure.

Next, the SOSU scheme was benchmarked against the RFS [14], [15] and the hybrid DDM [22] schemes in terms of PSNR vs bit rate performance of the reconstructed images. The left image was encoded using the DCT coding and reconstructed to yield $37 \mathrm{~dB}$. As mentioned before, for all the schemes the maximum number of input blocks used was set to 7 and the quality measure was allowed to vary from 26 to $36 \mathrm{~dB}$ in PSNR block. $_{\text {. For the }}$ SOSU scheme, two cases were considered. In the first case, only 64 input blocks were used, while the second case involved using

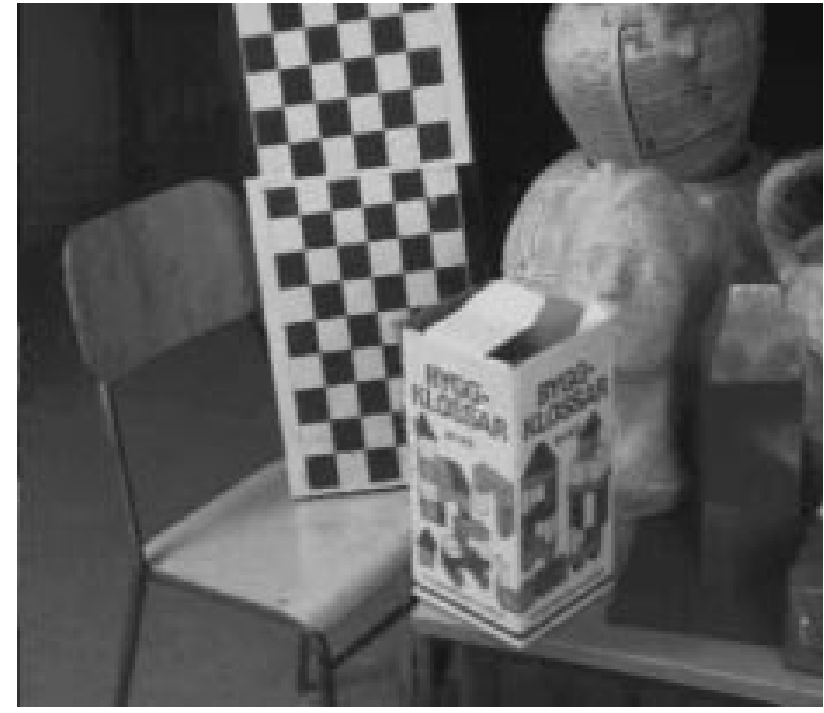

(a)

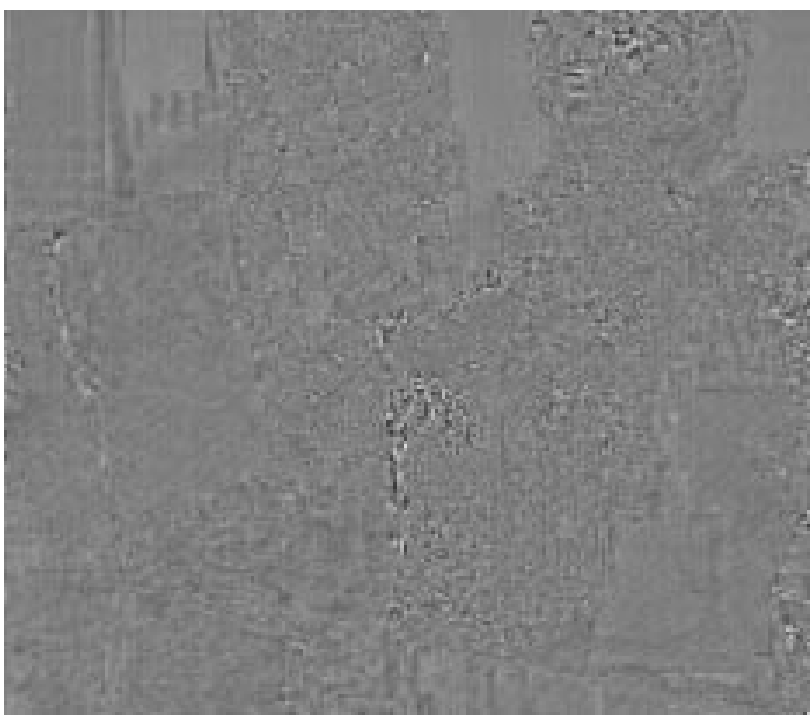

(b)

Fig. 8. Reconstructed and error images using the SOSU scheme with 64 image candidate blocks at 0.72 bpp for a stereo image pair "Chair." (a) Reconstructed image using SOSU scheme (33.78 dB, 0.72 bpp). (b) Error image using SOSU scheme.

not only the 64 input blocks but also all the 62 edge blocks as candidate basis vectors in $C$. The RFS scheme performs filtering on all the blocks inside the search region in order to estimate the disparity vector. The disparity vector is estimated by searching the spatial location at which the MSE between the estimated desired block, i.e., the filter output, and the desired block is minimized. The support region contained 16 blocks for a $4 \times 4$ filter order case. The input blocks were selected based upon the magnitude of their corresponding filter weights. The filter weights of the reduced-order filter were then re-estimated using the new input support and then used to estimate the desired block. An additional input block was included if the predefined quality measure was not satisfied [14]. Enlarging the support region does not necessarily improve the performance since the filter weights spread over a larger region. This makes it difficult to choose the best filter input block by just using the lag of the largest magnitude weight. The hybrid DDM scheme, on the other hand, es- 


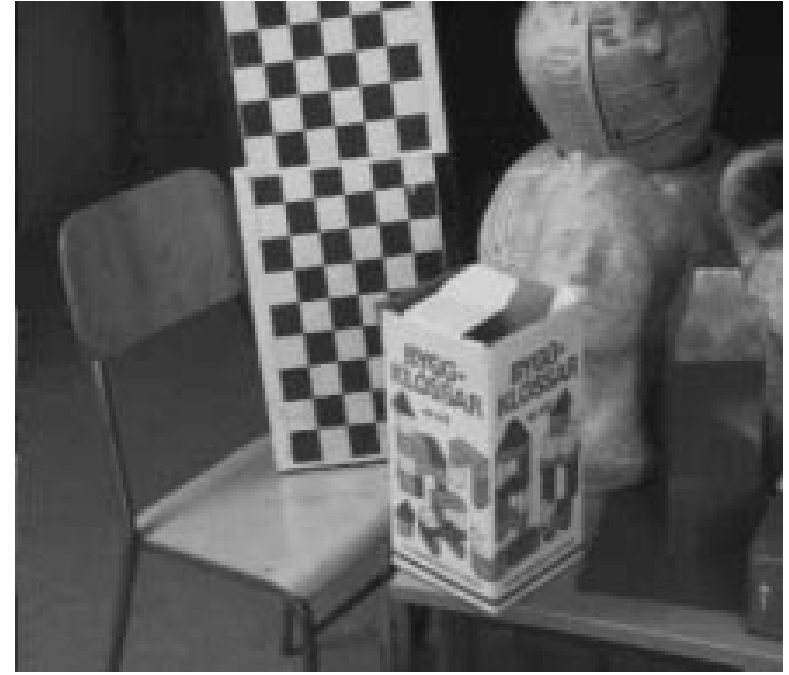

(a)

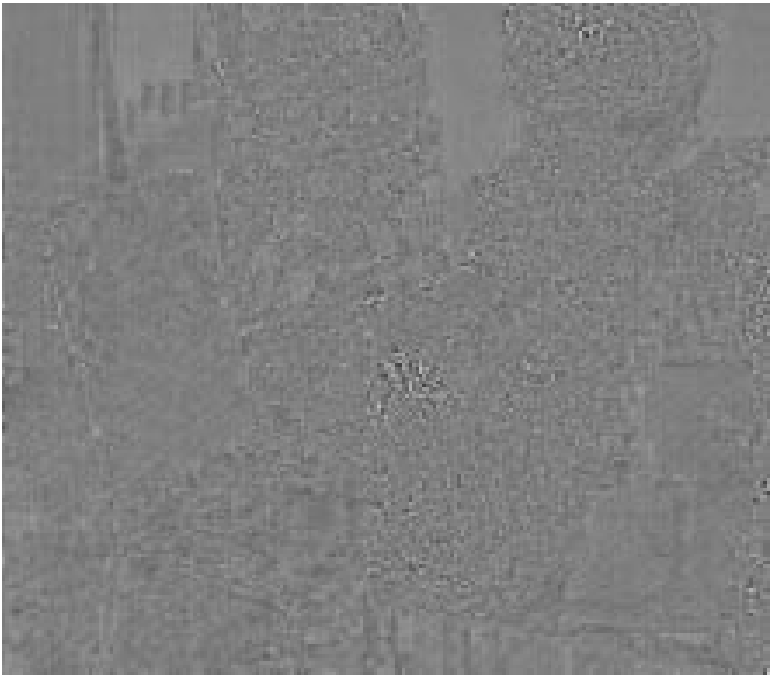

(b)

Fig. 9. Reconstructed and error images using the SOSU scheme with 64 image and 62 edge candidate blocks at 0.73 bpp for the stereo image pair "Chair." (a) Reconstructed image using the SOSU scheme $(34.50 \mathrm{~dB}, 0.73 \mathrm{bpp})$. (b) Error image using the SOSU scheme.

timates the disparity vector using the block-matching method. This scheme performs DCT encoding on the error vector after the disparity compensation; 64 DCT orthogonal basis vectors were used and the principal basis vectors were selected to reconstruct the desired block. The significance of each basis vector was determined according to the magnitude of its DCT coefficient.

As shown in Fig. 5(a), the performance of the SOSU scheme is substantially better than those of the RFS and hybrid DDM schemes in terms of PSNR versus bit rate. This is especially the case when both image blocks and edge blocks were used as candidate basis vectors. Fig. 5(b) provides the plot of the processing time in seconds versus bit rate. As can be seen, the RFS scheme requires considerable time for disparity estimation while the searching time for the best input blocks is relatively short. In contrast, the SOSU scheme requires relatively shorter time to estimate the disparity vector, but longer time to find the best input blocks. This is more evident as the bit rate is increased, which occurs when the number of input blocks needed to sat-

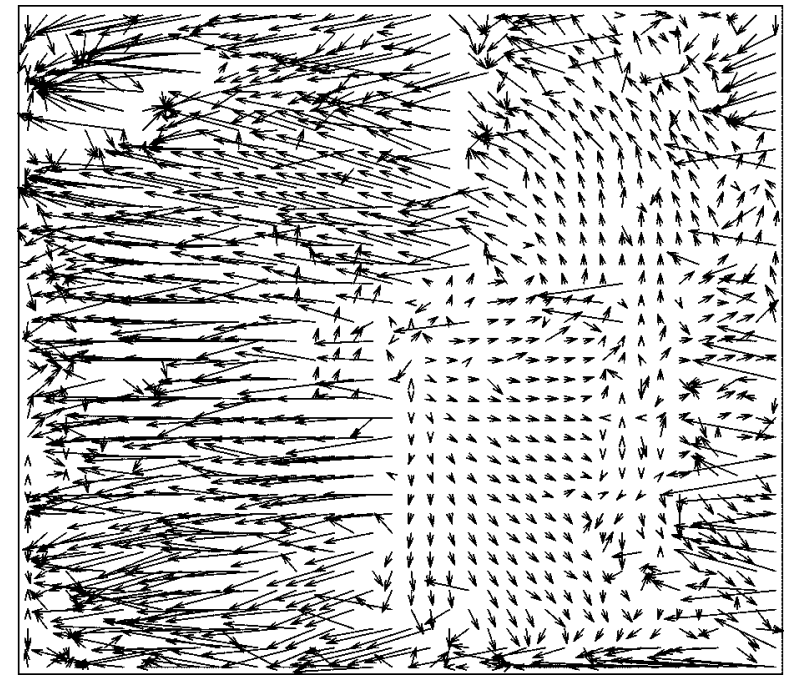

(a)

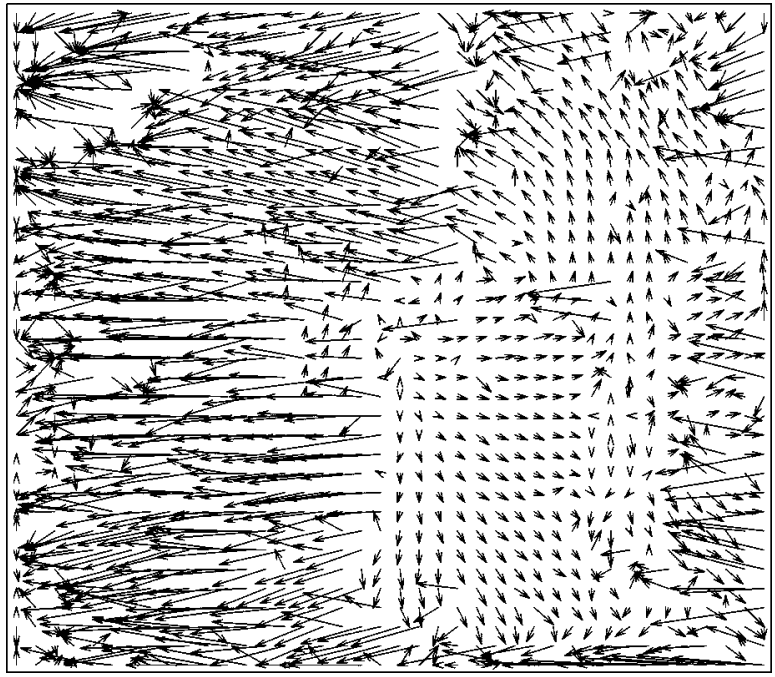

(b)

Fig. 10. Disparity field comparison of different schemes for the test stereo image pair "Chair." (a) Disparity field of the standard block-matching. (b) Disparity field of the SOSU method.

isfy the quality measure in the reconstructed image block is increased. The reason for this is that the proposed scheme uses more candidate input blocks than the reduced-order filtering scheme, and further, it requires orthogonalizing the remaining candidate input blocks at every stage. The computational time required for the hybrid DDM scheme is the smallest, since it uses fixed DCT basis vectors.

The difference or error images between the original right image and the reconstructed images for different schemes were also generated. Table I gives the values of maximum, minimum, and variance of the error image for different methods before the contrast mapping. The SOSU scheme using 64 image and 62 edge blocks provides the lowest residual variance compared to all the other schemes. The reconstructed and error images are shown in Figs. 6-9 for these different methods at approximately the same bit rate. The error images are then contrast enhanced to facilitate the visual comparison. The error image for the hybrid DDM scheme in Fig. 6(b) exhibits significant edge effects that are more prominent for the checkerboard 


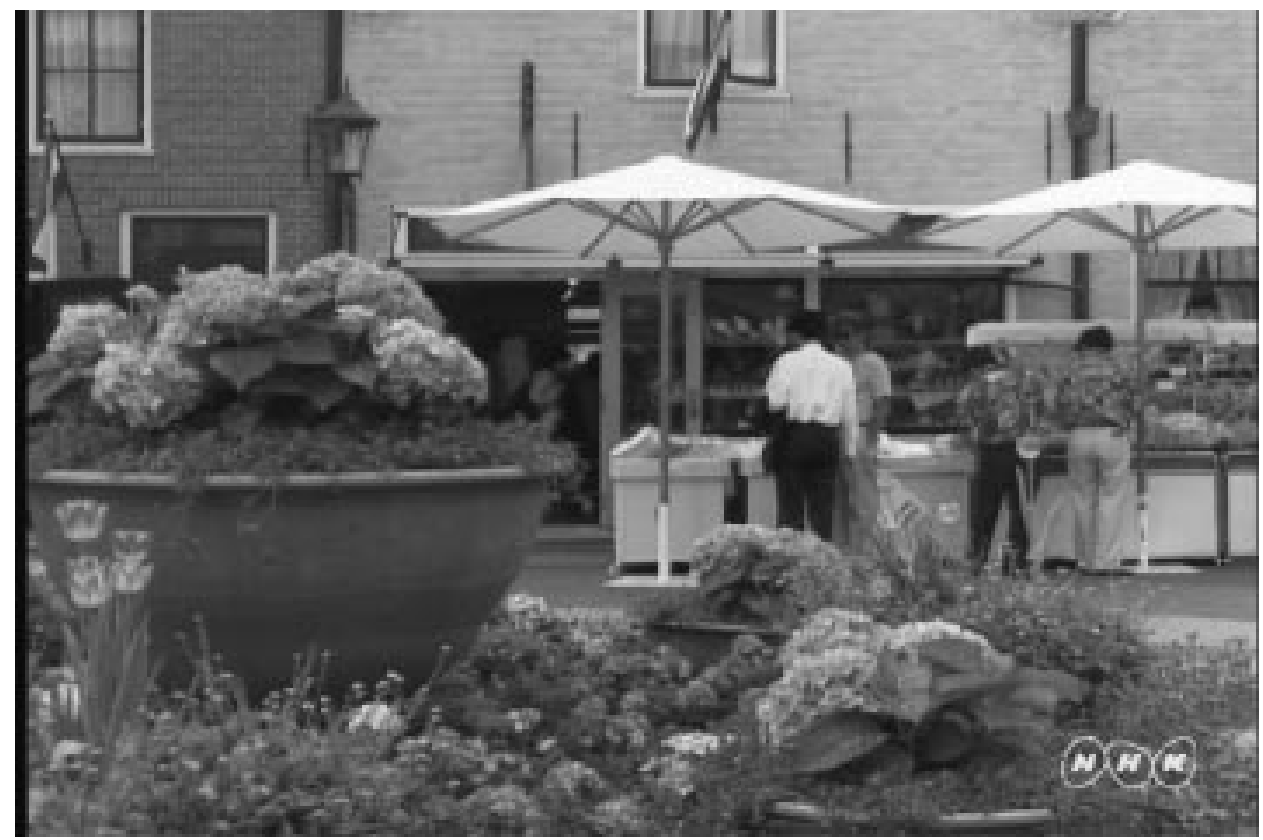

(a)

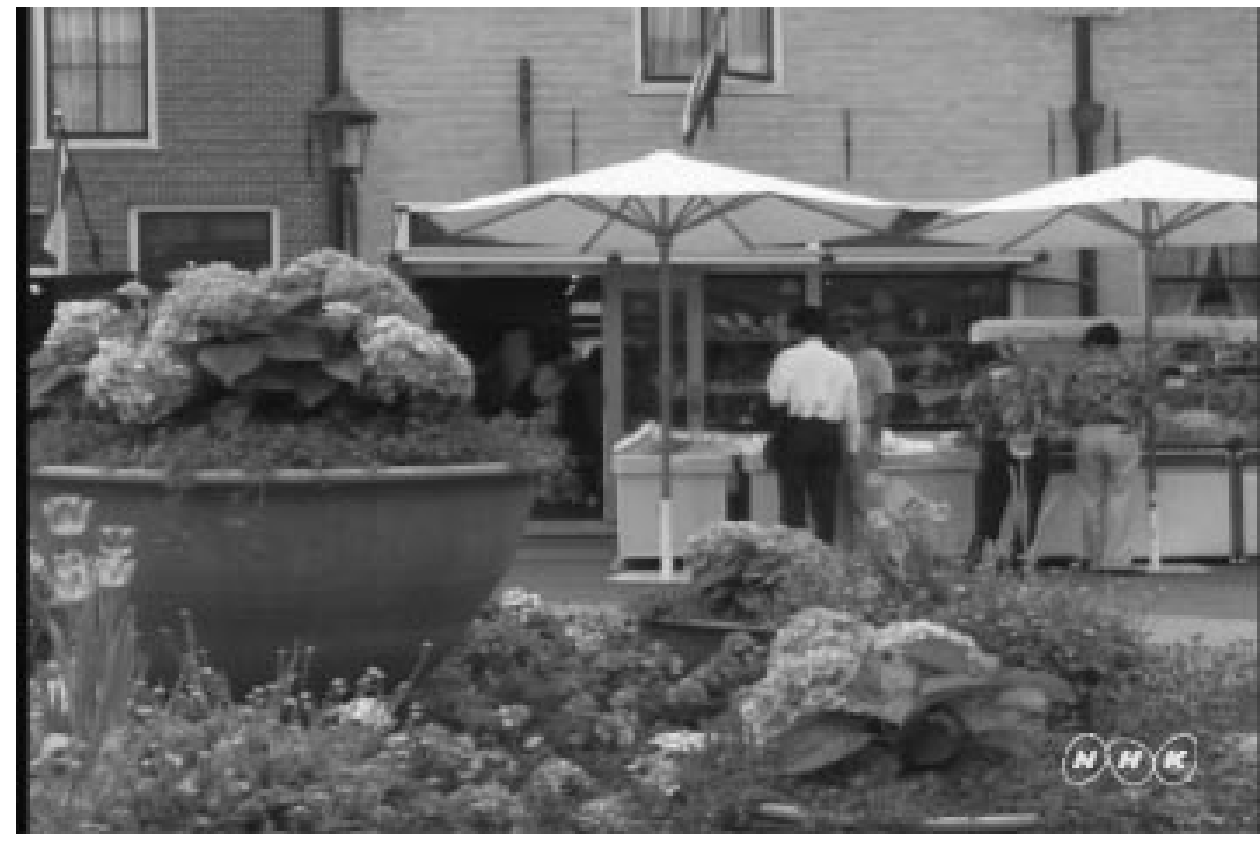

(b)

Fig. 11. Original real stereo image pair "Flowerpot," courtesy of NHK Corp. (a) Original left image. (b) Original right image.

pattern and in other areas with fine detail edges. The PSNR for the reconstructed image was measured to be $32.41 \mathrm{~dB}$ at 0.76 bpp. The RFS provides somewhat better compensation for certain areas while producing inferior results in the others, e.g., some of the blocks on the front of the container, as seen in Fig. 7(b). It should, however, be pointed out that contrast mapping to some extent exaggerates these effects. The PSNR for the image in Fig. 7(a) was found to be $31.81 \mathrm{~dB}$ at $0.76 \mathrm{bpp}$. The reconstructed and error images using the proposed SOSU scheme for the cases without and with the 62 edge blocks are shown in Figs. 8 and 9, respectively. The visual evaluation these error images clearly reveals the superior performance of this scheme. Most of the mismatching problems are removed as shown in the error image of Fig. 8(b) and more prominently in Fig. 9(b). In particular, the result in Fig. 9(b) is much better than those of the RFS and the hybrid DDM schemes. The PSNR for the reconstructed images in Figs. 8(a) and 9(a) were found to be 33.78 and $34.50 \mathrm{~dB}$ at 0.72 and $0.73 \mathrm{bpp}$, respectively.

Finally, the disparity field for the block-matching and SOSU filtering schemes were generated. As shown in Fig. 10(a) and (b), the disparity fields are not much different, since SOSU also relies on the block-matching initial estimates of the disparity vectors. It must be pointed out that the proposed filtering is mainly designed to provide compensation ability for the mismatching effects. However, it is possible to use our scheme for disparity estimation as long as certain modifications are incorporated. 


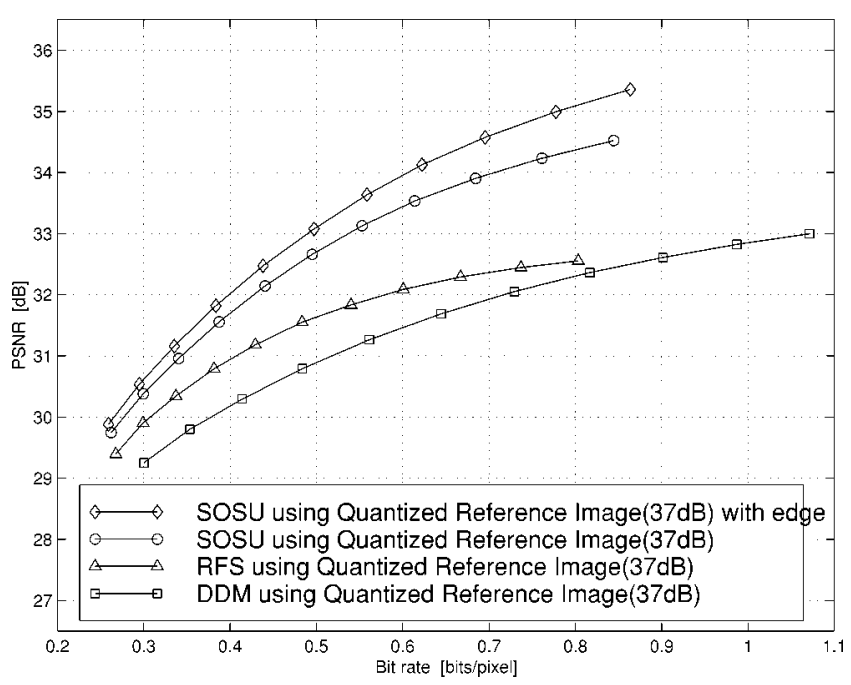

(a)

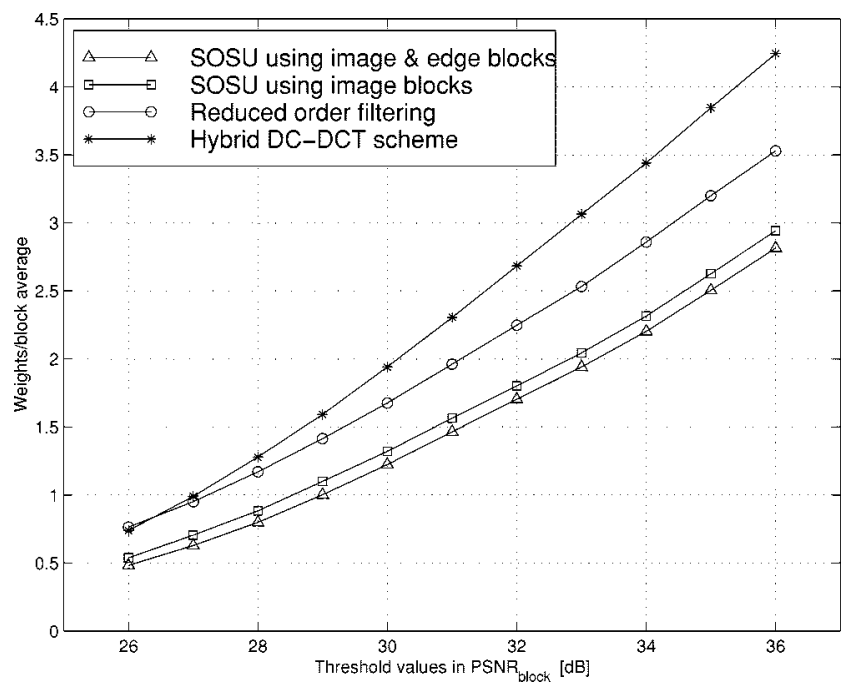

(b)

Fig. 12. Performance comparison of different schemes for the real stereo image pair "Flowerpot." (a) Performance comparison-PSNR versus bit rate. b) Performance comparison—average weights/block versus quality measures.

\section{B. Simulation Results on a Real Stereo Image Pair}

To evaluate the performance of the proposed SOSU scheme on real stereo images, a true 3DTV image pair was used. This image pair "Flowerpot" is shown in Fig. 11(a) and (b). Each image is of size $480 \times 720$ with 256 grey levels. The block size was chosen to be $8 \times 8$ and the search region was defined by extending the block coordinates 4 pixels to the left, 11 pixels to the right, 2 pixels to the top, and 1 pixel to the bottom margins. Comparing to the "Chair" image pair, for this image pair the distance between the two cameras is shorter hence leading to subtle disparity between the left and right images.

The same experiments as in the test stereo image pair "Chair" were conducted for this case. The performance of the different schemes was compared in terms of PSNR versus bit rate and average weights/block versus quality measure. Since the size of the search region is smaller in this case $(16 \times 4=64)$, only 6 bits are needed to encode the disparity vectors. All the other bit assignments are the same as in the previous case. As can be seen from Fig. 12(a), the improvement in the PSNR versus bit-rate
TABLE II

MaXimum, Minimum AND VARIANCE VALUeS OF THE ERROR IMAgES RECONSTRUCTED USING DIFFERENT SCHEMES FOR A STEREO IMAGE PAIR "FLOWERPOT"

\begin{tabular}{c||c|c|c}
\hline Schemes & Max. value & Min. value & Variance \\
\hline \hline Block-matching & 0.6078 & -0.8510 & 0.00250 \\
\hline Hybrid DC-DCT & 0.2997 & -0.2895 & 0.00062 \\
\hline Reduced order filtering & 0.4732 & -0.3632 & 0.00057 \\
\hline SOSU with image \& edge blocks & 0.2898 & -0.3124 & 0.00035 \\
\hline
\end{tabular}

performance for the SOSU scheme is substantially more evident on this real stereo image pair than the previous test image pair. The performance of the hybrid DDM scheme is inferior even to that of the RFS scheme. The reason being in contrast to the DDM scheme, the filtering scheme works well in compensating for the mismatching problems in regions with high spatial and textural activities. Fig. 12(b) shows the plot of the average weights/block versus different quality measure values PSNR $_{\text {block }}$ for the reconstruction using different schemes. It is interesting to note that the SOSU scheme requires lesser number of input blocks to satisfy the predefined quality measure than the RFS or hybrid DDM scheme. Another observation is that the average weights/block for the RFS scheme is less than that of the hybrid DDM scheme, even though the RFS scheme used 16 candidate input image blocks in its support region, while the hybrid DDM scheme used 64 basis vectors. This implies that comparing to the candidate input image blocks used in the RFS scheme and the SOSU scheme, the DCT basis vectors may not be appropriate to encode the residual image, especially in regions with high spatial activities.

The reconstructed and error images were also generated, as in the previous cases, to compare the visual appearance of the images. Table II gives the values of maximum, minimum, and variance of the error images for different schemes before the preprocessing process. As evident, the variance for the RFS scheme is slightly less than that of the hybrid DDM scheme, while the SOSU scheme using 64 image and 62 edge blocks offers the lowest variance compared to all the other schemes. This observation is consistent with the result obtained for the previous test stereo image pair. The reconstructed and error images are shown in Figs. 13-15 for these methods at approximately the same bit rate. Again, the error image for the hybrid DDM in Fig. 13(b) clearly exhibits edge effect problems throughout the image. The PSNR for the reconstructed image in Fig. 13(a) was measured to be $32.05 \mathrm{~dB}$ at $0.73 \mathrm{bpp}$. The RFS also suffers from edge effect problems more prominently in certain areas than the others, as shown in the error image in Fig. 14(b). The PSNR for the reconstructed image in Fig. 14(a) was found to be $32.43 \mathrm{~dB}$ at 0.74 bpp which shows a slight improvement over that of the hybrid DDM result. The reconstructed and error images using the proposed SOSU scheme for the case with 64 image blocks and 62 edge blocks are shown in Fig. 15(a) and (b), respectively. The visual evaluation of these images clearly demonstrates the superiority of the proposed scheme over the other schemes considered in this benchmark. As can be seen in Fig. 15(b), most of the edge effect problems are compensated for without sacrificing the bit rate performance. The results in Fig. 15(a) and (b) clearly show the effectiveness of the proposed SOSU. The PSNR for the reconstructed image in Fig. 15(a) was found to be 


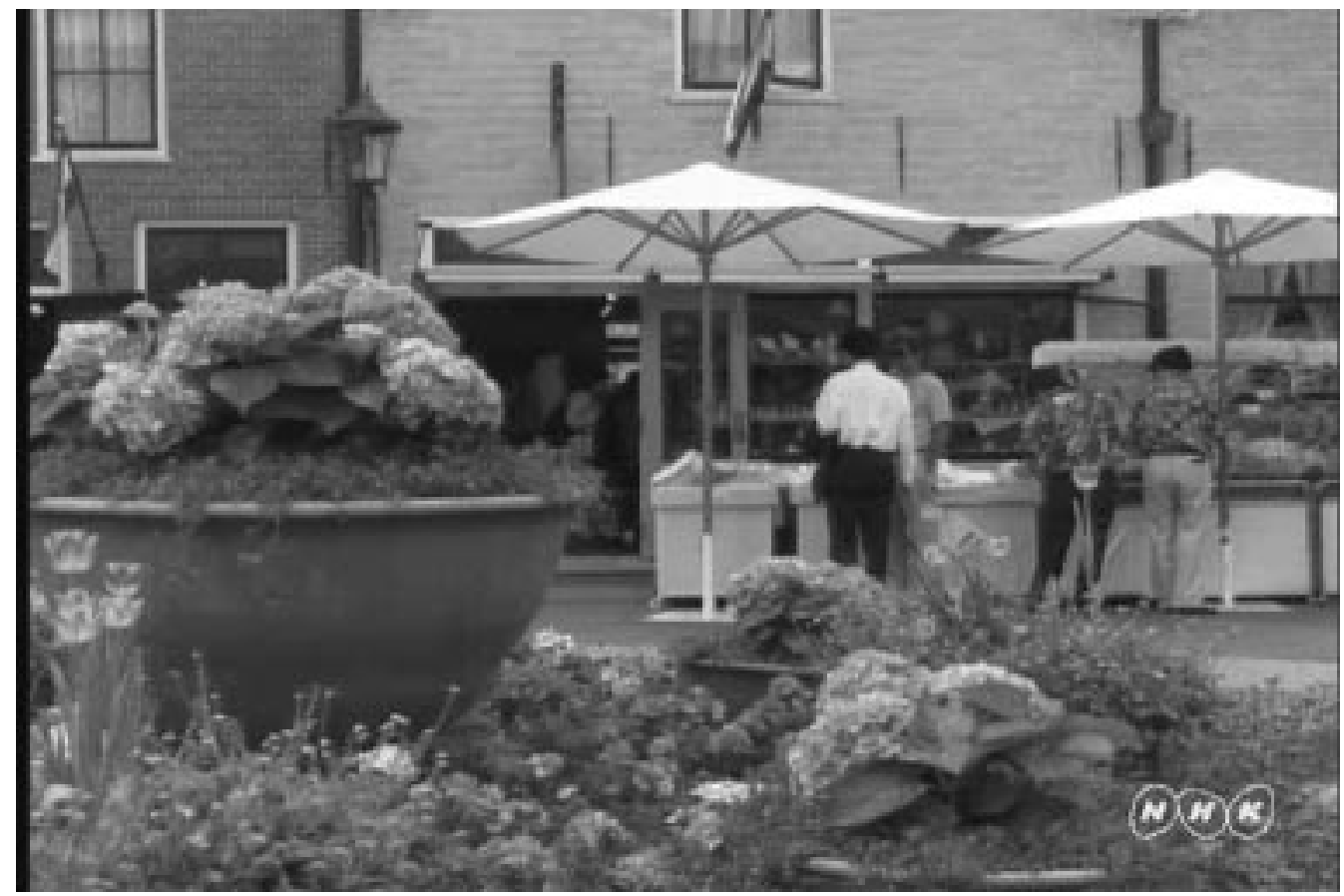

(a)

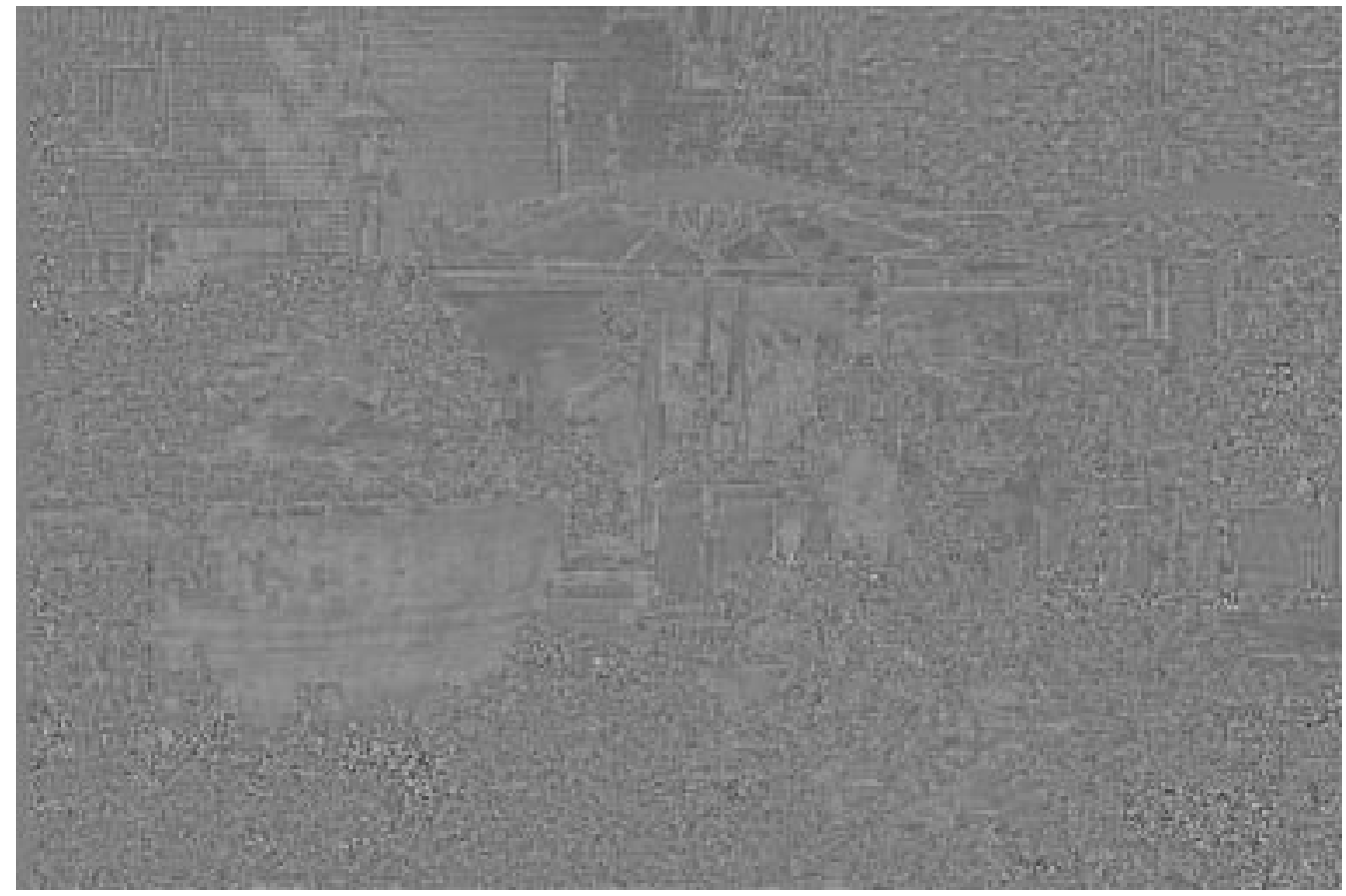

(b)

Fig. 13. Reconstructed and error images using the hybrid DC-DCT scheme at 0.73 bpp on the stereo image pair "Flowerpot." (a) Reconstructed image using the hybrid DC-DCT scheme (32.05 dB, 0.73 bpp). (b) Error image using the hybrid DC-DCT scheme.

$34.57 \mathrm{~dB}$ at $0.70 \mathrm{bpp}$, which indicates substantial improvement over those of the hybrid DDM and RFS schemes.

\section{CONCLUSION}

This paper presented a new 2-D filtering scheme for stereo image compression using the SOSU method. The method was inspired from the LS-based 2-D filtering scheme [14], [15], while circumventing its limitations and enhancing its compensation ability for the mismatching problems. Disparity estimation is performed using a simple block-matching scheme. To improve the compensation ability of the filtering after the disparity estimation process, the support region of the filter is expanded to include not only neighboring blocks but also 


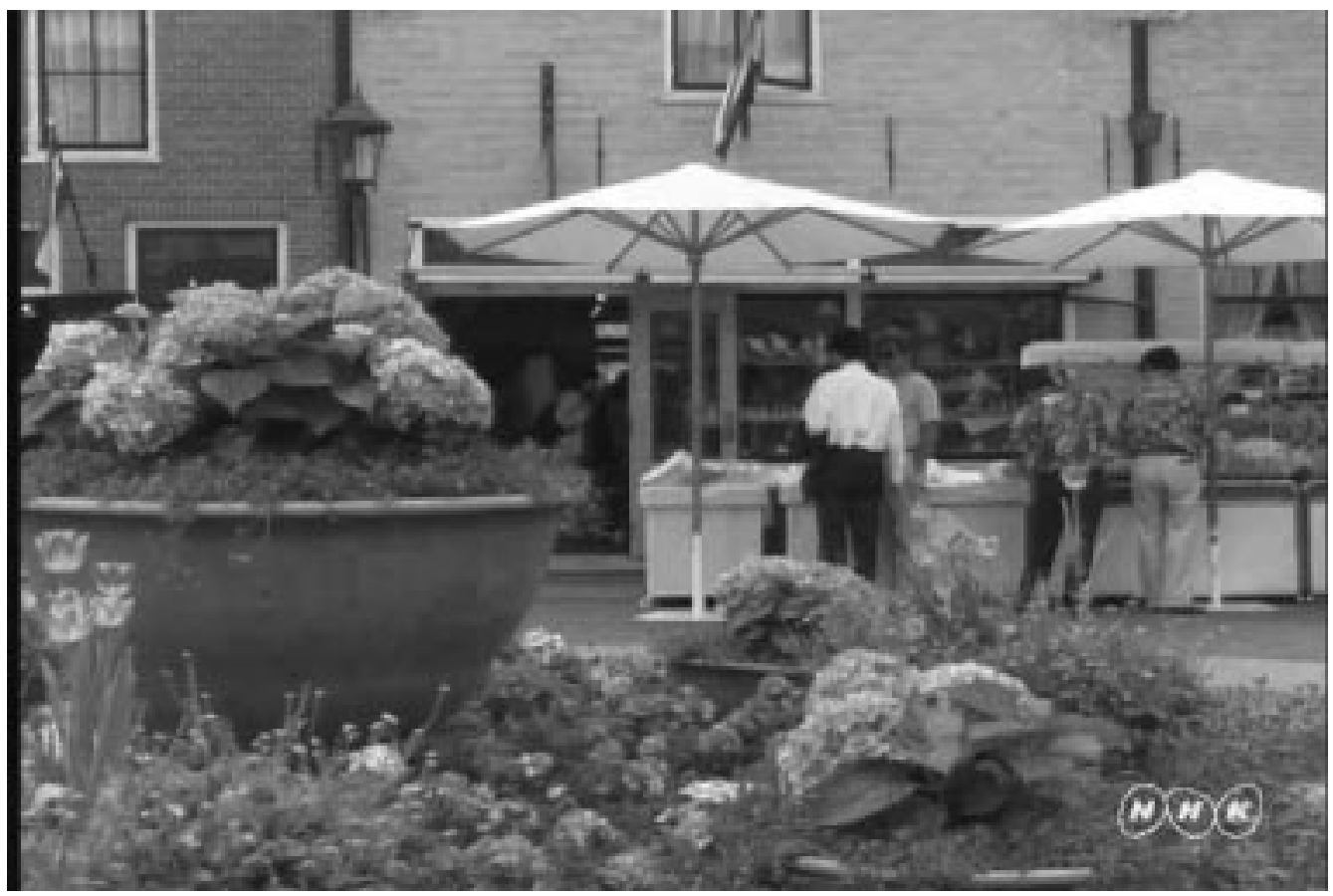

(a)

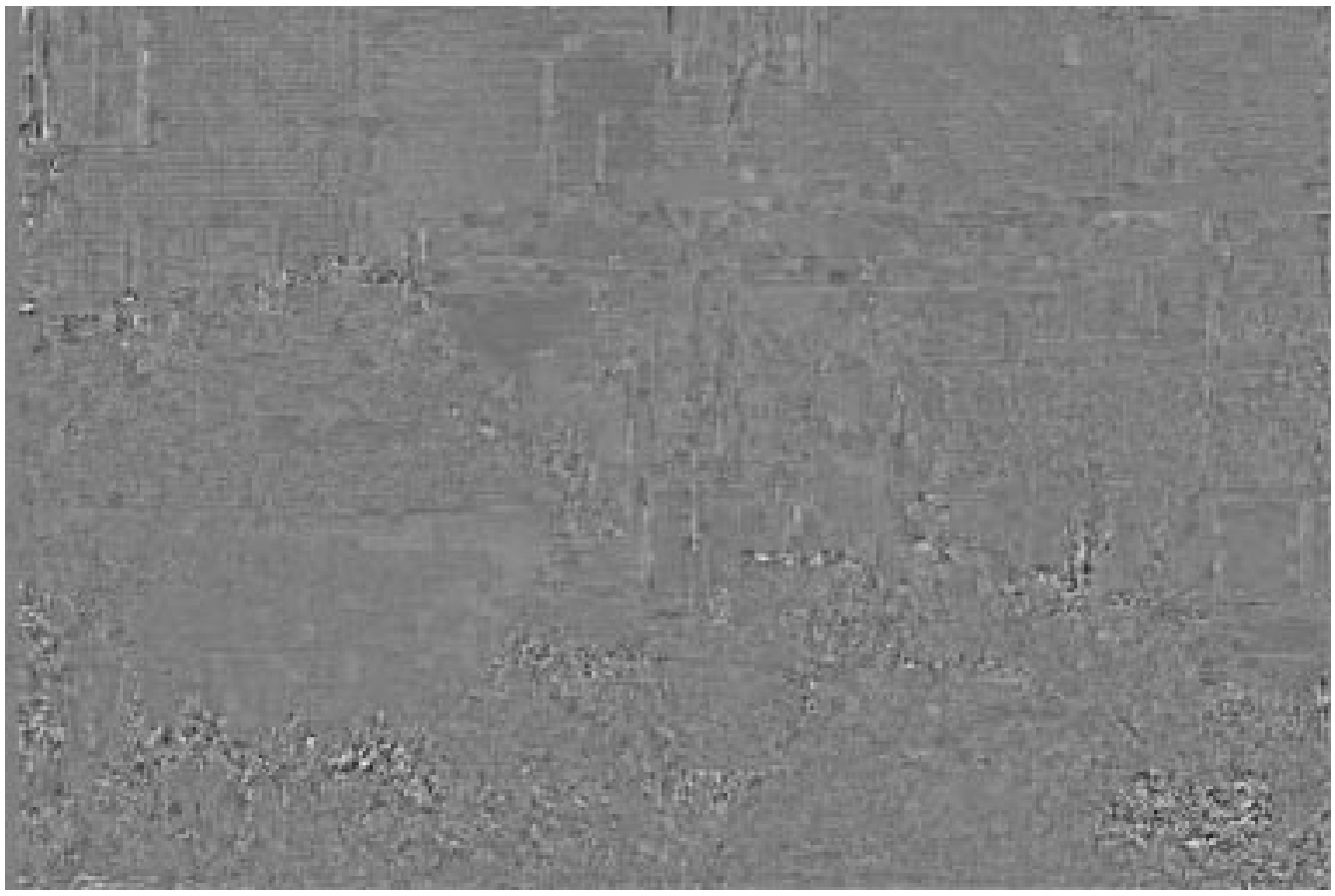

(b)

Fig. 14. Reconstructed and error images using the reduced-order filtering scheme at $0.74 \mathrm{bpp}$ for the stereo image pair "Flowerpot." (a) Reconstructed image using the reduced-order filtering scheme $(32.43 \mathrm{~dB}, 0.74 \mathrm{bpp})$. (b) Error image using the reduced-order scheme.

some typical edge blocks. This provides a more flexible and image-dependent representation and filtering. Consequently, the image subspace will contain a minimum number of best basis that satisfy a pre-selected fidelity measure for reconstructing the images in sequences of stereo images, while effectively removing the mismatching effects in these images. The proposed scheme orthogonalizes the input blocks in a sequential manner using the subspace projection updating. The operations can be carried out using simple vector inner product without any matrix manipulations. Simulation results of the SOSU demonstrated the excellent performance of this scheme when compared to those of the LS-based 2-D filtering and hybrid DC-DCT schemes for compensating for the effects of mismatching, and improving the PSNR and bit rates in stereo image compression applications. Overall, this scheme is found to be very promising for stereo image/video compression ap- 


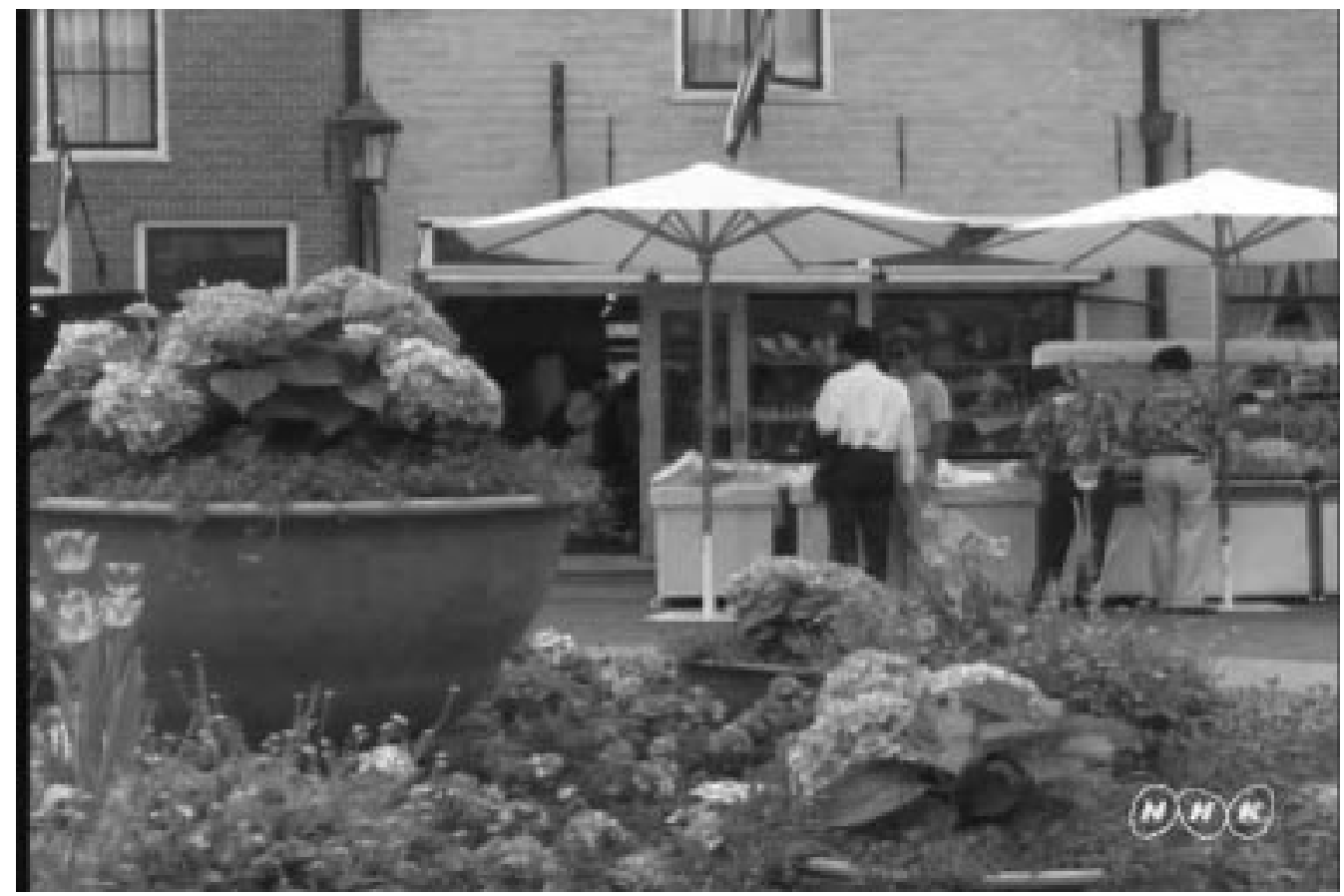

(a)

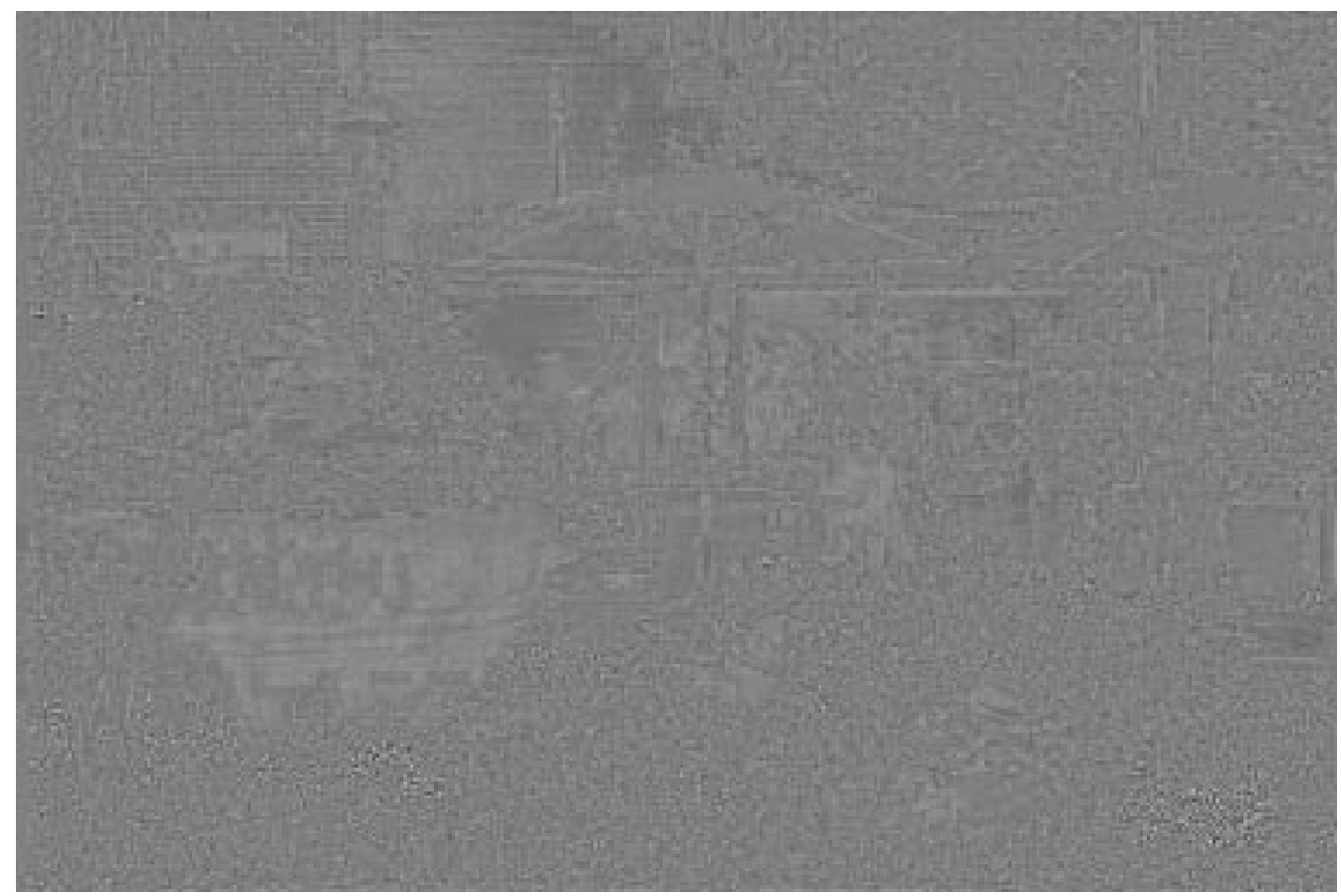

(b)

Fig. 15. Reconstructed and error images using the SOSU scheme with the subspace of 64 image and 62 edge candidate input blocks at 0.7 bpp for the stereo image pair "Flowerpot." (a) Reconstructed image using the SOSU scheme (35.57 dB, 0.7 bpp). (b) Error image using the SOSU scheme.

plications since not only it provides high quality reconstructed images with great compensation for mismatching problems, but also is ideally suitable for parallel implementation using array processors owing to its block-based nature. Future study should include finding the optimal edge subspace to improve the compensation ability as well as extending the proposed scheme on stereo image sequences to evaluate its real-life applicability in the 3DTV area.

\section{ACKNOWLEDGMENT}

The authors would like to thank Dr. T. Chen and NHK Corporation for providing one of the image pairs used in this paper.

\section{REFERENCES}

[1] T. Motoki, H. Isono, and I. Yuyama, "Present state of three-dimensional television research," Proc. IEEE, vol. 83, pp. 1009-1021, July 1995. 
[2] D. Tzovaras, M. G. Strintzis, and H. Sanhinoglou, "Evaluation of multiresolution block matching technique for motion and disparity estimation," Signal Processing: Image Commun., vol. 6, pp. 59-67, 1994.

[3] D. J. LeGall, "MPEG: A video compression standard for multimedia applications," Commun. ACM, vol. 34, pp. 946-958, 1991.

[4] Video Coding for Low Bit Rate Communication, May 1996. ITU-T Recommendation H.263.

[5] V. E. Seferidis and D. V. Papadimitriou, "Improved disparity estimation in srereoscopic television,” Electron. Lett., vol. 29, no. 9, pp. 782-783, 1993.

[6] S. Sethuraman, A. G. Jordan, and M. W. Siegel, "A multiresolutional region based segmentation scheme for stereoscopic image compression," SPIE, vol. 2419, pp. 265-274, 1995.

[7] H. Aydinoglu and M. H. Hayes, III, "Stereo image coding," IEEE Int. Symp. Circuits and Systems, pp. 247-250, 1995.

[8] H. Aydinoglu and M. H. Hayes, "Multi-view image coding using local orthogonal bases," Visual Commun. Image Processing, 1997.

[9] S. Mallat and Z. Zhang, "Matching pursuits with time-frequency dictionaries," IEEE Trans. Signal Processing, vol. 41, pp. 3397-3415, Dec. 1993.

[10] R. Neff and A. Zakhor, "Very low bit-rate video coding based on matching pursuits," IEEE Trans. Circuits Syste. Video Technol., vol. 7, pp. 158-171, Feb. 1997.

[11] M. R. Banham and J. C. Brailean, "A selective update approach to matching pursuits video coding," IEEE Trans. Circuits Syst. Video Technol., vol. 7, pp. 119-129, Feb. 1997.

[12] B. H. Juang and A. H. Gray, "Multiple stage vector quantization for speech coding," in Proc. ICASSP'82, vol. 1, Apr. 1982, pp. 597-600.

[13] A. Gersho and R. M. Gray, Vector Quantization and Signal Compression. Boston, MA: Kluwer, 1991.

[14] S. H. Seo, M. R. Azimi-Sadjadi, and B. Tian, "A least-squares-based 2-D filtering scheme for stereo image compression," IEEE Trans. Image Processing, vol. 9, pp. 1967-1972, Nov. 2000

[15] S. H. Seo, "2-D Filter-Based Disparity Estimation and Compensation Schemes for Stereo Image Compression," Colorado State Univ., Fort Collins, CO, 1998.

[16] G. Strang, Linear Algebra and Its Applications. Orlando, FL: Harcourt Brace Jovanovich, 1988

[17] B. Ramamurthi and A. Gersho, "Clasified vector quantization of images," IEEE Trans. Commun., vol. COM-34, no. 11, pp. 1105-1115, Nov. 1986.

[18] J. M. Cioffi and T. Kailath, "Fast recursive-least-squares transversal filters for adaptive filtering," IEEE Trans. Acoust. Speech, Signal Processing, vol. ASSP-32, pp. 304-338, Apr. 1984.

[19] S. T. Alexander, Adaptive Signal Processing: Theory and Applications: Springer-Verlag, 1986.

[20] S. Haykin, Adaptive Filter Theory. Englewood Cliffs, NJ: PrenticeHall, 1995.
[21] J. M. Cioffi, "Fast Transversal Filter Applications for Communications Applications," Ph.D. dissertation, Stanford Univ., Stanford, CA, 1984.

[22] K. R. Rao and J. J. Hwang, Techniques and Standards for Image, Video and Audio Coding. Englewood Cliffs, NJ: Prentice-Hall, 1996.

[23] A. K. Jain, Fundamentals of Digital Image Processing. Englewood Cliffs, NJ: Prentice-Hall, 1989.

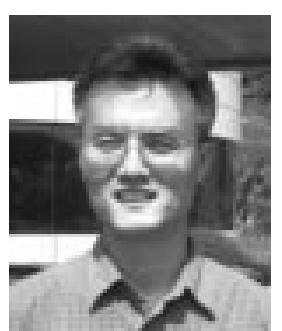

Sang-Hoon Seo received the B.S. degree in electronic engineering from Kyunghee University, Seoul, Korea, in 1985, the M.S. degree in electrical and electronic engineering from the Korea Advanced Institute of Science and Technology (KAIST), Seoul Korea, in 1988, and the Ph.D. degree in electrical engineering from Colorado State University, Fort Collins, in 1998

Since 1988, he has been with the Radio Research Laboratory, Seoul, Korea. His research interests are in the areas of communications and digital signal processing with applications to stereo video systems, voice coding, and signal enhancement.

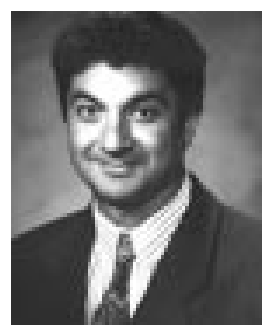

Mahmood R. Azimi-Sadjadi (S'81-M'81-SM'89) received the M.S. and Ph.D. degrees from Imperial College of Science and Technology, University of London, London, U.K., in 1978 and 1982, respectively, both in electrical engineering with specialization in digital signal/image processing.

$\mathrm{He}$ is currently a Full Professor at the Electrical and Computer Engineering Department, Colorado State University (CSU), Fort Colllins, in addition to being Director of the Multi-sensory Computing Laboratory (MUSCL) and Digital Signal/Image Laboratory at CSU. His main areas of interest include digital signal and image processing, target detection, classification and tracking, adaptive filtering and system identification, and neural networks. His research efforts in these areas have produced over 120 journal and refereed conference publications. He is the co-author of the book Digital Filtering in One and Two Dimensions (New York: Plenum, 1989).

Dr. Azimi-Sadjadi served as an Associate Editor of the IEEE TRANSACTIONS ON SIGNAL PROCESSING and is currently serving as an Associate Editor of IEEE TRANSACTIONS ON NEURAL NETWORKS. He is the recipient of the 1999 ABELL Teaching Award, the 1993 ASEE-Navy Senior Faculty Fellowship Award, the 1991 CSU Dean's Council Award, and the 1984 DOW Chemical Outstanding Young Faculty Award. 